JOURNAL OF THE

AMERICAN MATHEMATICAL SOCIETY

Volume 16, Number 2, Pages 393-426

S 0894-0347(02)00411-3

Article electronically published on November 26, 2002

\title{
ON THE EQUATION $\operatorname{div} Y=f$ AND APPLICATION TO CONTROL OF PHASES
}

\author{
JEAN BOURGAIN AND HAÏM BREZIS
}

\section{INTRODUCTION}

The purpose of this paper is to present new results concerning the equation

$$
\operatorname{div} Y=f \quad \text { on } \mathbb{T}^{d},
$$

i.e., we work on $\mathbb{R}^{d}$ with $2 \pi$-periodic functions in all variables. In what follows we will always assume that $d \geq 2$ and that

$$
\int_{Q} f=0
$$

where $Q=(0,2 \pi)^{d}$. The notations $L^{p}, W^{1, p}$, etc. refer to $L^{p}\left(\mathbb{T}^{d}\right), W^{1, p}\left(\mathbb{T}^{d}\right)$, etc. or to $2 \pi$-periodic functions in $L_{l o c}^{p}\left(\mathbb{R}^{d}\right), W_{l o c}^{1, p}\left(\mathbb{R}^{d}\right)$, etc. We denote by $L_{\#}^{p}$ the space of functions in $L^{p}$ satisfying (1.2).

Clearly, (1.1) is an underdetermined problem which admits many solutions. A standard way of tackling (1.1) is to look for a vector field $Y$ satisfying the additional condition

$$
\text { curl } Y=0 \text {, }
$$

i.e., one looks for a special $Y$ of the form

$$
Y=\operatorname{grad} u .
$$

Equation (1.1) then becomes

$$
\Delta u=f
$$

and the standard $L^{p}$-regularity theory yields a solution $u \in W^{2, p}$ when $f \in L_{\#}^{p}, 1<$ $p<\infty$. Consequently (1.1) has a solution $Y \in W^{1, p}$ for every $f \in L_{\#}^{p}, 1<p<\infty$. More precisely, the operator div : $W^{1, p} \rightarrow L_{\#}^{p}$ admits a right inverse which is a bounded linear operator $K: L_{\#}^{p} \rightarrow W^{1, p}$. Strictly speaking, we should write $Y \in\left(W^{1, p}\right)^{d}\left(=d\right.$-fold copy of $\left.W^{1, p}\right)$, div $:\left(W^{1, p}\right)^{d} \rightarrow L^{p}$, etc. But we will often omit the superscript $d$ to alleviate notation.

Three limiting cases are of interest:

Received by the editors January 14, 2002 and, in revised form, October 2, 2002.

2000 Mathematics Subject Classification. Primary 35C99, 35F05, 35F15, 42B05, $46 \mathrm{E} 35$

Key words and phrases. Divergence equations, gradient equations, critical Sobolev norms.

The first author was partially supported by NSF Grant DMS-9801013.

The second author was partially sponsored by a European Grant ERB FMRX CT98 0201. He is also a member of the Institut Universitaire de France.

The authors thank C. Fefferman, P. Lax, P. Mironescu, L. Nirenberg, T. Rivière, M. Vogelius and D. Ye for useful comments. 
Case 1: $\mathbf{p}=\mathbf{1}$. It is well known that when $f \in L^{1}$ equation (1.3) does not necessarily admit a solution $u \in W^{2,1}$. However, one might still hope to have some solution $Y$ of (1.1) in $W^{1,1}$ or at least in $B V$. This is not true: for some $f$ 's in $L^{1}$, equation (1.1) has no solution in $B V$ and not even in $L^{d /(d-1)}$; see Section 2.1.

Case 2: $\mathbf{p}=\infty$. It is well known that when $f \in L^{\infty}$ equation (1.3) does not necessarily admit a solution $u \in W^{2, \infty}$. However, one might hope to find a solution $Y$ of (1.1) in $W^{1, \infty}$. This is not true: McMullen [13] has shown that for some $f$ 's in $L^{\infty}$ (even continuous $f$ ) equation (1.1) has no solution in $W^{1, \infty}$. This is proved using a duality argument and a "non-estimate" of Ornstein [16]; see Section 2.2.

Case 3: $\mathbf{p}=\mathbf{d}$. This is the heart of our work. For every $f \in L_{\#}^{d}$, equation (1.3) admits a solution $u \in W^{2, d}$ and thus equation (1.1) admits a solution $Y=\operatorname{grad} u \in$ $W^{1, d}$. Since $W^{1, d}$ is not contained in $L^{\infty}$ (this is a limiting case for the Sobolev imbedding), we cannot assert that this $Y$ belongs to $L^{\infty}$. In fact, we give in Section 3 (Remark 7) an explicit $f \in L^{d}$ such that the corresponding $Y=\operatorname{grad} u$ does $n o t$ belong to $L^{\infty}$. However one might still hope that given any $f \in L_{\#}^{d}$ there is some $Y \in L^{\infty}$ solving (1.1). This is indeed true:

Proposition 1. Given any $f \in L_{\#}^{d}$ there exists some $Y \in L^{\infty}$ solving (1.1) (in the sense of distributions) with

$$
\|Y\|_{L^{\infty}} \leq C(d)\|f\|_{L^{d}}
$$

Remark 1. A more precise statement established in the course of the proof says that there exists $Y \in C^{0}$ satisfying (1.1) and (1.4).

The proof of Proposition 1 is quite elementary; see Section 3. It relies on the Sobolev-Nirenberg imbedding $W^{1,1} \subset L^{d /(d-1)}$ (and even $B V \subset L^{d /(d-1)}$ ) combined with duality, i.e., Hahn-Banach. As a consequence, the argument is not constructive, and $Y$ is not obtained as above via a bounded linear operator acting on $f$. In fact, surprisingly, the operator div has no bounded right inverse in this setting:

Proposition 2. There exists no bounded linear operator $K: L_{\#}^{d} \rightarrow L^{\infty}$ such that $\operatorname{div} K f=f \quad \forall f \in L_{\#}^{d}$ (in the sense of distributions).

Remark 2. Another way of formulating Proposition 2 is to say that the subspace $\left\{Y \in L^{\infty} ; \operatorname{div} Y=0\right\}$ admits no complement in the space $\left\{Y \in L^{\infty} ; \operatorname{div} Y \in\right.$ $\left.L^{d}\right\}$ equipped with its natural norm. Alternatively, the closed subspace grad $u$; $\left.u \in W^{1,1}\right\}$ has no complement in $L^{1}$; see Section 3 .

To summarize: for every $f \in L_{\#}^{d}$, equation (1.1) admits

a) a solution $Y_{1} \in W^{1, d}$,

b) a solution $Y_{2} \in L^{\infty}$.

A natural question is whether there exists a solution $Y$ of $(1.1)$ in $L^{\infty} \cap W^{1, d}$. This is indeed one of our main results.

Theorem 1. For every $f \in L_{\#}^{d}$ there exists a solution $Y \in L^{\infty} \cap W^{1, d}$ of (1.1) satisfying

$$
\|Y\|_{L^{\infty}}+\|Y\|_{W^{1, d}} \leq C(d)\|f\|_{L^{d}} .
$$

Despite the simplicity of this statement the argument is rather involved and a simpler proof would be desirable.

We will present two techniques to tackle Theorem 1. 
First proof of Theorem 1 when $d=2$ (see Section 4). It relies on HahnBanach (via duality) and thus it is not constructive. But it is rather elementary; the main ingredient is the new estimate (1.6) which is established by $L^{2}$-Fourier methods.

Lemma 1. On $\mathbb{T}^{2}$ we have

$$
\left\|u-\int u\right\|_{L^{2}} \leq C\|\operatorname{grad} u\|_{L^{1}+H^{-1}}, \quad \forall u \in L^{2},
$$

for some absolute constant $C$.

The main difficulty, in proving (1.6), stems from the fact that if we decompose

$$
\operatorname{grad} u=h_{1}+h_{2}
$$

with $h_{1} \in L^{1}$ and $h_{2} \in H^{-1}$, then $h_{1}$ and $h_{2}$ need not be gradients themselves; it is only their sum which is a gradient.

The analogue of Lemma 1 for $d>2$ is the estimate on $\mathbb{T}^{d}$,

$$
\left\|u-\int u\right\|_{L^{d /(d-1)}} \leq C(d)\|\operatorname{grad} u\|_{L^{1}+W^{-1, d /(d-1)}} .
$$

We have no direct proof of (1.7). But it can be deduced by duality from the statement of Theorem 1 (and thus from the second proof presented in Section 7).

Second proof of Theorem 1, valid for all $d \geq 2$ (see Sections 5 and 6). We exhibit via a constructive (nonlinear) argument some explicit $Y \in W^{1, d} \cap L^{\infty}$ satisfying (1.1) and (1.5). The argument for $d=2$ is simpler and we start with this case for expository reasons.

One should observe a certain analogy with the Fefferman-Stein [10] decomposition of BMO-functions and Uchiyama's 21] constructive proof. Indeed, returning to equation (1.1) and defining $F$ by $|\xi| \hat{F}(\xi)=\hat{f}(\xi)$, we obtain that $F \in W^{1, d} \subset B M O$ and (1.1) becomes

$$
F=\sum_{j=1}^{d} R_{j} Y_{j}
$$

with $R_{j}=j^{\text {th }}$ Riesz transform $\left(\widehat{R_{j} \psi}(\xi)=\hat{\psi}(\xi) \frac{\xi_{j}}{|\xi|}\right), Y=\left(Y_{1}, \ldots, Y_{d}\right)$.

The statement of Theorem 1 is that (1.8) has a solution $Y \in L^{\infty} \cap W^{1, d}$. Recall that according to Fefferman-Stein [10] any $F \in B M O$ has a decomposition of the form

$$
F=Y_{0}+\sum_{j=1}^{d} R_{j} Y_{j} \quad \text { with } Y_{0}, Y_{1}, \ldots, Y_{d} \in L^{\infty} .
$$

The proof of this decomposition is again by duality and nonconstructive. The later constructive approach from Uchiyama 21 gives a different proof of (1.9). If we assume moreover that $F \in W^{1, d}$, Uchiyama's argument gives that (1.9) has a solution $Y_{0}, Y_{1}, \ldots, Y_{d} \in L^{\infty} \cap W^{1, d}$. The new result in this paper shows that, in fact, for $F \in W^{1, d}$, the $Y_{0}$-component is unnecessary and (1.8) holds for some $Y_{1}, \ldots, Y_{d} \in L^{\infty} \cap W^{1, d}$.

It should be mentioned that to achieve our decomposition we do use significantly different methods from Uchiyama. This raises the question what are the function 
spaces $X, W^{1, d} \subset X \subset B M O$, such that every $F \in X$ has a decomposition

$$
F=\sum_{j=1}^{d} R_{j} Y_{j}
$$

where $Y_{j} \in L^{\infty}$ or (assuming the Riesz transforms bounded on $X$ ) the stronger property $Y_{j} \in L^{\infty} \cap X$.

Remark 3. Using Theorem 1 we will prove (in Sections 4 and 6) that a slightly stronger conclusion holds:

Theorem 1'. For every $f \in L_{\#}^{d}$ there exists a solution $Y \in C^{0} \cap W^{1, d}$ of (1.1) satisfying (1.5).

The original motivation for studying (1.1) comes from the following question about lifting discussed in Bourgain-Brezis-Mironescu [3], 44, [5]. Consider the equation

$$
g=e^{i \varphi} \quad \text { on } \mathbb{T}^{d}
$$

where $\varphi$ is a smooth real-valued function.

Question. Assuming $g$ is controlled in $H^{1 / 2}$, what kind of estimate can we deduce for $\varphi$ ?

Here is a first easy consequence of Theorem 1.

Corollary 1. We have

$$
\left\|\varphi-\int \varphi\right\|_{L^{d /(d-1)}} \leq C(d)\left(1+\|g\|_{H^{1 / 2}}\right)\|g\|_{H^{1 / 2}} .
$$

Proof. Write

$$
\operatorname{grad} g=i e^{i \varphi} \operatorname{grad} \varphi
$$

and thus

$$
\operatorname{grad} \varphi=-i \bar{g}(\operatorname{grad} g)
$$

Multiplying by $Y$ gives

$$
\int_{Q} \varphi \operatorname{div} Y=\int_{Q} i \bar{g} Y \cdot \operatorname{grad} g .
$$

Given $f \in L^{d}$ we obtain from Theorem 1 some $Y$ satisfying (1.1) (with $f$ replaced by $\left.f-\int f\right)$ and (1.5). Thus we have

$$
\left|\int\left(\varphi-\int \varphi\right) f\right| \leq\|g\|_{H^{1 / 2}}\left(\|\bar{g} Y\|_{H^{1 / 2}}\right) .
$$

But

$$
\begin{aligned}
& \|\bar{g} Y\|_{H^{1 / 2}} \leq\|g\|_{H^{1 / 2}}\|Y\|_{L^{\infty}}+\|g\|_{L^{\infty}}\|Y\|_{H^{1 / 2}} \\
& (\text { by }(1.5)) \leq C\left(\|g\|_{H^{1 / 2}}\|f\|_{L^{d}}+\|f\|_{L^{d}}\right)
\end{aligned}
$$

where we have used the obvious fact that $\|Y\|_{H^{1 / 2}} \leq C\|Y\|_{W^{1, d}}$. Combining (1.14) and (1.15) yields (1.11).

Remark 4. Estimate (1.11) cannot be improved, replacing the norm \|\|$_{L^{d /(d-1)}}$ by \|\|$_{L^{p}}, p>d /(d-1)$. This may be seen by choosing $g=e^{i \varphi}$ with $\varphi(x)=$ $\left(|x|^{2}+\varepsilon^{2}\right)^{-\alpha / 2}$ with $\alpha<d-1, \alpha$ close to $(d-1)$ and $\varepsilon$ close to 0 (the same example has already been used in Bourgain-Brezis-Mironescu [3], Lemma 5). There is however a better estimate than (1.11), namely 
Theorem 4. Let $\varphi$ be a smooth real-valued function on $\mathbb{T}^{d}$ and set $g=e^{i \varphi}$, then

$$
\|\varphi\|_{H^{1 / 2}+W^{1,1}} \leq C(d)\left(1+\|g\|_{H^{1 / 2}}\right)\|g\|_{H^{1 / 2}} .
$$

Theorem 4 has been announced in Bourgain-Brezis-Mironescu [4] (Theorem 3) and is proved in Section 8. Our proof of Theorem 4 is a direct estimate based on paraproducts. In view of the preceding argument one may wonder whether Theorem 4 can be proved by solving a divergence equation. After duality the required statement would be

$$
\left\|u-\int u\right\|_{H^{1 / 2}+W^{1,1}} \leq C\|\operatorname{grad} u\|_{H^{-1 / 2}+L^{1}}
$$

but we do not know whether (1.16) holds.

We now turn to the question of coupling equation (1.1) with the Dirichlet condition

$$
Y=0 \quad \text { on } \partial Q .
$$

This question was addressed (in various forms) by a few authors; see e.g. ArnoldScott-Vogelius [2, Duvaut-Lions [9] (Theorem 3.2), X. Wang 22], Temam [20] (Proposition 1.2(ii) and Lemma 2.4) and the references therein to MagenesStampacchia [12] and Nečas [14]. Our aim is to establish the analogue of Theorem $1^{\prime}$ under the Dirichlet condition. We start with the following known fact (see e.g. Arnold-Scott-Vogelius [2] for $d=2$ ).

Theorem 2. Given $f \in L_{\#}^{p}(Q), 1<p<\infty$, there exists some $Y \in W_{0}^{1, p}(Q)$ satisfying (1.1) with

$$
\|Y\|_{W^{1, p}} \leq C(p)\|f\|_{L^{p}} .
$$

Moreover $Y$ can be chosen, depending linearly on $f$.

The operator and the estimate do not depend on $p$ assuming we stay away from the end points.

For the convenience of the reader we include a new proof; our technique is extremely elementary and can be adapted to establish, for the limiting case $p=d$,

Theorem 3. Given $f \in L_{\#}^{d}(Q)$ there exists some $Y \in C^{0}(\bar{Q}) \cap W_{0}^{1, d}(Q)$ satisfying (1.1) with

$$
\|Y\|_{L^{\infty}}+\|Y\|_{W^{1, d}} \leq C\|f\|_{L^{d}} .
$$

Theorem 3 is stronger than Theorem $1^{\prime}$. However it will be deduced from Theorem $1^{\prime}$. There are variants of Theorems 2 and 3 when $Q$ is replaced by a Lipschitz domain in $\mathbb{R}^{d}$ (see Section 7.2).

The plan of the paper is the following:

1. Introduction.

2. The cases $f \in L^{p}$ with $p=1$ and $p=\infty$.

3. Proofs of Propositions 1 and 2 and related questions.

4. Proof of Theorem 1 when $d=2$ via duality.

5. Proof of Theorem 1 when $d=2$ (explicit construction).

6. Proof of Theorem 1 when $d>2$ (explicit construction).

7. The equation $\operatorname{div} Y=f$ with Dirichlet condition. Proof of Theorems 2 and 3.

8. Estimation of the phase in $H^{1 / 2}+W^{1,1}$. Proof of Theorem 4. 


\section{The CASES $f \in L^{p}$ With $p=1$ AND $p=\infty$}

We consider here equation (1.1) with $f \in L_{\#}^{p}$ and ask whether there exists a solution $Y \in W^{1, p}$ of (1.1) when $p=1$ and $p=\infty$. As we have already mentioned in the Introduction the answer is negative. Here is the proof.

2.1. The case $p=1$. Assume by contradiction that for every $f \in L_{\#}^{1}$ there is some $Y \in W^{1,1}$ satisfying (1.1). It follows that the linear operator

$$
T u=\operatorname{div} u \text { from } E=W^{1,1} \text { into } F=L_{\#}^{1}
$$

is bounded and surjective. By the open mapping principle there is a constant $C$ such that for every $f \in F$ there exists a solution $Y \in E$ of (1.1) satisfying

$$
\|Y\|_{W^{1,1}} \leq C\|f\|_{L^{1}} .
$$

We now use a duality argument which occurs frequently in the rest of the paper. We will deduce that $W^{1, d} \subset L^{\infty}$ with continuous injection, and since this is false, we infer that for some $f$ 's in $F$ there is no $Y \in W^{1,1}$ satisfying (1.1).

Let $u \in W^{1, d}$ and set

$$
\operatorname{grad} u=h \in L^{d} .
$$

Given any $f \in L^{1}$, let $Y \in W^{1,1}$ be such that

$$
\operatorname{div} Y=f-\int f
$$

and

$$
\|Y\|_{W^{1,1}} \leq C\left\|f-\int f\right\|_{L^{1}}
$$

Taking the scalar product of (2.1) with $Y$ and integrating yields

$$
\int_{Q}\left(u-\int_{Q} u\right) f=-\int_{Q} h Y .
$$

Consequently

$$
\left|\int_{Q}\left(u-\int_{Q} u\right) f\right| \leq\|h\|_{L^{d}}\|Y\|_{L^{d /(d-1)}} .
$$

By the Sobolev-Nirenberg imbedding we have $W^{1,1} \subset L^{d /(d-1)}$ and thus

$$
\|Y\|_{L^{d /(d-1)}} \leq C\|Y\|_{W^{1,1}} \leq C\|f\|_{L^{1}} .
$$

Combining (2.2) and (2.3) we deduce that $\left(u-\int_{Q} u\right) \in L^{\infty}$ with

$$
\left\|u-\int_{Q} u\right\|_{L^{\infty}} \leq C\|\operatorname{grad} u\|_{L^{d}}
$$

Impossible.

Remark 5. The same argument shows that equation (1.1) with $f \in L_{\#}^{1}$ need not have a solution $Y$ in the sense of distributions with $Y \in L^{d /(d-1)}$. (Note, however, that the solution $Y$ given via (1.3) belongs to $L^{p}, \forall p<d /(d-1)$, and even to weak- $\left.L^{d /(d-1)}\right)$. It suffices to follow the above argument with $E=W^{1,1}$ replaced by

$$
\widetilde{E}=\left\{Y \in L^{d /(d-1)} ; \operatorname{div} Y \in L^{1}\right\}
$$

equipped with its natural norm. 
2.2. The case $p=\infty$. This case has been settled negatively by McMullen [13] (the interest in this kind of problem grew out of the study of the equation $\operatorname{det}(\nabla \varphi)=f$ with $\varphi$ bi-Lipschitz and also from a question of Gromov [11] on separated nets; see Dacorogna-Moser [18], Ye [24], Rivière-Ye [17, [18], Burago-Kleiner [7]).

For the convenience of the reader we sketch a proof when $d=2$, which is essentially similar to the one of McMullen 13. We argue by contradiction as above. Then, for every $f \in L^{\infty}$ there is a $Y \in W^{1, \infty}$ satisfying

$$
\operatorname{div} Y=f-\int f
$$

and

$$
\|Y\|_{W^{1, \infty}} \leq C\|f\|_{L^{\infty}}
$$

Let $\psi$ be a smooth function on $\mathbb{T}^{2}$ and set $g=\psi_{x_{1} x_{2}}$. Write

$$
\int g_{x_{1}} Y_{1}+g_{x_{2}} Y_{2}=-\int g f=-\int \psi_{x_{1} x_{1}} Y_{1 x_{2}}+\psi_{x_{2} x_{2}} Y_{2 x_{1}} .
$$

Consequently

$$
\left|\int g f\right| \leq C\left(\left\|\psi_{x_{1} x_{1}}\right\|_{L^{1}}+\left\|\psi_{x_{2} x_{2}}\right\|_{L^{1}}\right)\|f\|_{L^{\infty}}
$$

and thus

$$
\|g\|_{L^{1}}=\left\|\psi_{x_{1} x_{2}}\right\|_{L^{1}} \leq C\left(\left\|\psi_{x_{1} x_{1}}\right\|_{L^{1}}+\left\|\psi_{x_{2} x_{2}}\right\|_{L^{1}}\right) .
$$

This contradicts a celebrated "non-inequality" of Ornstein [16] and completes the proof.

Remark 6. The same argument shows that equation (1.1) with $f \in C^{0}$ and $\int f=0$ need not have a solution $Y \in W^{1, \infty}$.

\section{Proofs of Propositions 1 and 2 and Related questions}

Proof of Proposition 1. Recall the Sobolev-Nirenberg imbedding $W^{1,1} \subset L^{d /(d-1)}$ and, more generally, $B V \subset L^{d /(d-1)}$ with

$$
\left\|u-\int u\right\|_{L^{d /(d-1)}} \leq C(d)\|\operatorname{grad} u\|_{\mathcal{M}} \quad \forall u \in B V,
$$

where $\mathcal{M}$ denotes the space of measures. Set

$$
E=C^{0}, \quad F=L_{\#}^{d}
$$

and consider the unbounded linear operator $A=D(A) \subset E \rightarrow F$, defined by

$$
D(A)=\left\{Y \in E ; \operatorname{div} Y \in L^{d}\right\}, \quad A Y=\operatorname{div} Y,
$$

so that $A$ is densely defined and has closed graph. Clearly we have

$$
\begin{aligned}
E^{*} & =\mathcal{M}, \quad F^{*}=L_{\#}^{d /(d-1)}, \\
D\left(A^{*}\right) & =F^{*} \cap B V, \quad A^{*} u=\operatorname{grad} u .
\end{aligned}
$$

By (3.1) we have

$$
\|u\|_{F^{*}} \leq C(d)\left\|A^{*} u\right\|_{E^{*}} \quad \forall u \in D\left(A^{*}\right) .
$$

It follows from the closed-range theorem (see e.g. Brezis [6], Section II.7) that $A$ is surjective. More precisely, we claim that for any $f \in F$ there is some $Y \in E$ satisfying (1.1) and

$$
\|Y\|_{L^{\infty}} \leq 2 C(d)\|f\|_{L^{d}},
$$

where $C(d)$ is the constant in (3.1). 
Indeed, let $f \in F$ with $\|f\|_{L^{d}}=1$ and consider the two convex sets

$$
B=\left\{Y \in E ;\|Y\|_{E}<2 C(d)\right\}
$$

and

$$
L=\{Y \in E ; \operatorname{div} Y=f\} .
$$

We have to prove that $B \cap L \neq \emptyset$. Suppose not, and $B \cap L=\emptyset$. Then, by Hahn-Banach there exists $\mu \in E^{*}, \mu \neq 0$, and $\alpha \in \mathbb{R}$ such that

$$
\langle\mu, Y\rangle \leq \alpha \quad \forall Y \in B
$$

and

$$
\langle\mu, Y\rangle \geq \alpha \quad \forall Y \in L .
$$

From (3.2) we have $\|\mu\| \leq \alpha / 2 C(d)$ and from (3.3) we deduce, in particular, that $\langle\mu, Z\rangle=0 \quad \forall Z \in N(A)$. It follows that $\mu \in N(A)^{\perp}=R\left(A^{*}\right)$. Hence there exists some $u \in F^{*} \cap B V$ such that $\operatorname{grad} u=\mu$. Applying (3.1) we see that

$$
\|u\|_{L^{d /(d-1)}} \leq C(d)\|\mu\| \leq \alpha / 2
$$

On the other hand, by $(3.3), \forall Y \in L$,

$$
\alpha \leq\langle\mu, Y\rangle=\langle\operatorname{grad} u, Y\rangle=-\int u \operatorname{div} Y=-\int u f \leq\|u\|_{L^{d /(d-1)}} \leq \alpha / 2 .
$$

This is impossible since $\alpha>0$ (because $\mu \neq 0$ ).

Remark 7. The special solution of (1.1) given by $Y=\operatorname{grad} u$, where $u$ is the solution of (1.3), belongs to $W^{1, d}$ when $f \in L^{d}$; however, in general, it does not belong to $L^{\infty}$. Here is an example due to L. Nirenberg. Using $\left(x_{1}, x_{2}, \ldots, x_{d}\right)$ as coordinates in $\mathbb{R}^{d}$ consider the function

$$
u=x_{1}|\log r|^{\alpha} \zeta
$$

where $\zeta$ is a smooth cut-off function with support near 0 and $0<\alpha<(d-1) / d$. Note that $Y=\operatorname{grad} u$ does not belong to $L^{\infty}$ while

$$
|\Delta u| \leq \frac{C}{r}|\log r|^{\alpha-1}
$$

so that $\Delta u \in L^{d}$.

We now turn to the proof of Proposition 2, i.e., the non-existence of a bounded right inverse $K: L_{\#}^{d} \rightarrow L^{\infty}$ for the operator div. We present two proofs. The first is the simplest: after a standard averaging trick we obtain a bounded multiplier $L^{d} \rightarrow L^{\infty}$ and we reach a contradiction by a direct summability consideration. The second proof is related to Remark 2: the existence of $K$ would yield a factorization of the identity map $I: W^{1,1} \rightarrow L^{d /(d-1)}$ through the Banach space $L^{1}$; however no such factorization exists by a general argument from the geometry of Banach spaces.

First proof of Proposition 2. Assume $K: L_{\#}^{d} \rightarrow L^{\infty}$ is a bounded operator satisfying $\operatorname{div} K=I$ on $L_{\#}^{d}$. Then the averaged operator

$$
\widetilde{K}=\int_{\mathbb{T}^{d}} \tau_{-x} K \tau_{x} d x
$$

where $\tau_{x} f(y)=f(y+x)$, still satisfies

$$
\operatorname{div} \widetilde{K}=I \quad \text { on } L^{d} .
$$


On the other hand, $\widetilde{K}$ is clearly a multiplier

$$
\widetilde{K}\left(e^{i n \cdot x}\right)=\left(\lambda_{1}(n), \lambda_{2}(n), \ldots, \lambda_{d}(n)\right) e^{i n \cdot x}
$$

which is bounded from $L^{d}$ into $L^{\infty}$ and hence from $L^{1}$ into $L^{d^{\prime}}$ where $d^{\prime}=d /(d-1)$. By (3.5) we have

$$
\sum_{j=1}^{d} n_{j} \lambda_{j}(n)=1 \quad \forall n \in \mathbb{Z}^{d}
$$

so that

$$
|\lambda(n)|^{2}=\sum_{j=1}^{d}\left|\lambda_{j}(n)\right|^{2} \geq 1 /|n|^{2} \quad \forall n .
$$

Consider the multiplier

$$
M\left(e^{i n \cdot x}\right)=\frac{1}{|n|^{\frac{d}{2}-1}} e^{i n \cdot x}, \quad n \neq 0 .
$$

Then $M$ is bounded from $L^{d^{\prime}}$ into $L^{2}$. Hence $M \widetilde{K}$ is a bounded multiplier from $L^{1}$ into $L^{2}$. Thus

$$
\sum_{\substack{n \in \mathbb{Z}^{d} \\ n \neq 0}} \frac{\left|\lambda_{j}(n)\right|^{2}}{|n|^{d-2}}<\infty, \quad \forall j
$$

Summing over $j=1,2, \ldots, d$, and using (3.6) we deduce

$$
\sum_{\substack{n \in \mathbb{Z}^{d} \\ n \neq 0}} \frac{1}{|n|^{d}}<\infty
$$

A contradiction.

Second proof of Proposition 2. Assuming the existence of $K: L_{\#}^{d} \rightarrow L^{\infty}$ we obtain a factorization of the identity map $I: W^{1,1} \rightarrow L^{d^{\prime}}$ as

$$
I=K^{*} \circ \operatorname{grad}
$$

which, in particular, gives a factorization of $I$ through the Banach space $L^{1}$. We claim that there in no such factorization, as a consequence of Grothendieck's theorem on absolutely summing operators. Both the result and the method are well known and we briefly recall them (see Wojtaszczyk 23] for details). First take $d=2$. Then $I: W^{1,1} \rightarrow L^{2}$ and we consider the operator $I \circ D$ where $D: L^{2} \rightarrow W^{1,1}$ is defined by

$$
D\left(e^{i n \cdot x}\right)=\frac{1}{\sqrt{1+|n|^{2}}} e^{i n \cdot x} .
$$

Thus $D$ is clearly bounded as an operator into $H^{1}$, hence into $W^{1,1}$. Since $I$ is assumed to factor through $L^{1}$, so does $I \circ D$ : 


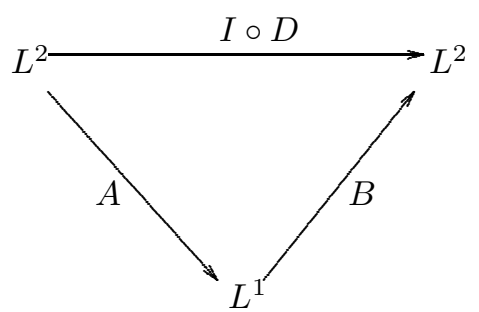

Next, recall Grothendieck's theorem that any bounded operator $B: L^{1} \rightarrow L^{2}$ is 1-summing, i.e.,

$$
\pi_{1}(B) \equiv \sup \left\{\sum\left\|B x_{i}\right\| ;\left(x_{i}\right) \subset L^{1} \text { and } \max _{x^{*} \in L^{\infty},\left\|x^{*}\right\| \leq 1} \sum\left|\left\langle x_{i}, x^{*}\right\rangle\right| \leq 1\right\} \leq K_{G}\|B\|,
$$

where $K_{G}$ is Grothendieck's constant.

From the usual ideal properties, we obtain

$$
\begin{array}{r}
\left(\sum_{n \in \mathbb{Z}^{2}} \frac{1}{1+|n|^{2}}\right)^{1 / 2}=\|I \circ D\|_{H S}=\pi_{2}(I \circ D) \leq \pi_{1}(I \circ D) \\
=\pi_{1}(B \circ A) \leq\|A\| \pi_{1}(B) \leq K_{G}\|A\|\|B\|<\infty,
\end{array}
$$

which in an obvious contradiction.

For $d>2$, we have $I: W^{1,1} \rightarrow L^{d^{\prime}}$ and we consider the multiplication operator $M: L^{d^{\prime}} \rightarrow L^{2}$ given by $M\left(e^{i n \cdot x}\right)=(1+|n|)^{1-\frac{d}{2}} e^{i n \cdot x}$. Hence, considering now $M \circ I \circ D: L^{2} \rightarrow L^{2}$ factoring through $L^{1}$, we obtain a contradiction again:

$$
\left(\sum \frac{1}{(1+|n|)^{d-2}\left(1+|n|^{2}\right)}\right)^{1 / 2}=\|M \circ I \circ D\|_{H S}=\pi_{2}(M \circ I \circ D) \leq \pi_{1}(M \circ I \circ D)<\infty .
$$

Proof of Remark 2. Consider the Banach space

$$
E=\left\{Y \in L^{\infty} ; \operatorname{div} Y \in L^{d}\right\}
$$

equipped with its natural norm $\|Y\|_{L^{\infty}}+\|\operatorname{div} Y\|_{L^{d}}$. Then

$$
N=\left\{Y \in L^{\infty} ; \operatorname{div} Y=0\right\}
$$

is a closed subspace of $E$ which admits no complement in $E$. Indeed, set

$$
F=L_{\#}^{d}
$$

and consider the bounded linear operator $T: E \rightarrow F$ defined by $T Y=\operatorname{div} Y$. By Proposition 1, $T$ is surjective. If $N=N(T)$ admits a complement in $E$, then $T$ has a bounded right inverse, i.e., an operator $S: F \rightarrow E$ such that

$$
\operatorname{div}(S f)=f \quad \forall f \in F
$$

(see e.g. Brezis [6], Théorème II.10). But this is impossible by Proposition 2.

Similarly, the subspace

$$
R=\left\{\operatorname{grad} u ; u \in W^{1,1}\right\}
$$

of $L^{1}$ is closed and admits no complement in $L^{1}$. Indeed, consider the spaces $E=\left\{u \in W^{1,1} ; \int u=0\right\}, F=L^{1}$ and the operator $T=\operatorname{grad}$, a bounded linear injective operator from $E$ into $F$. If $R=R(T)$ admits a complement in $F$, then 
$T$ has a bounded left inverse $S: F \rightarrow E$ (see e.g. Brezis [6], Théorème II.11). In particular, $S: F \rightarrow L_{\#}^{d /(d-1)}$ satisfies

$$
S(\operatorname{grad} u)=u, \quad \forall u \in W^{1,1} \text { with } \int u=0 .
$$

Then $S^{*}: L_{\#}^{d} \rightarrow L^{\infty}$ satisfies

$$
\operatorname{div}\left(S^{*} f\right)=f, \quad \forall f \in L_{\#}^{d},
$$

and this is again impossible by Proposition 2 .

\section{Proof of Theorem 1 When $d=2$ via Duality}

We now return to the periodic setting and we will prove the slightly stronger form of Theorem 1 ,

Theorem $\mathbf{1}^{\prime}$ (for $d=2$ ). For every $f \in L_{\#}^{2}$ there exists a solution $Y \in C^{0} \cap H^{1}$ of (1.1) with

$$
\|Y\|_{L^{\infty}}+\|Y\|_{H^{1}} \leq C\|f\|_{L^{2}}
$$

for some absolute constant $C$.

Theorem $1^{\prime}$ is proved by duality from

Lemma 2. On $\mathbb{T}^{2}$ we have

$$
\left\|u-\int u\right\|_{L^{2}} \leq C\|\operatorname{grad} u\|_{L^{1}+H^{-1}}, \forall u \in L^{2}
$$

where $C$ is an absolute constant.

Assuming the lemma we turn to the

Proof of Theorem $1^{\prime}$. First observe that

$$
L^{1}+H^{-1} \subset \mathcal{M}+H^{-1}
$$

and that

$$
\|\cdots\|_{L^{1}+H^{-1}}=\|\cdots\|_{\mathcal{M}+H^{-1}} \text { on } L^{1}+H^{-1}
$$

(this may be easily seen using regularization by convolution).

Let $E=C^{0} \cap H^{1}, F=L_{\#}^{2}$ and consider the bounded operator $T: E \rightarrow F$ defined by $T Y=\operatorname{div} Y$. Clearly, $T^{*}: F^{*}=F \rightarrow E^{*}=\mathcal{M}+H^{-1}$ is given by $T^{*} u=\operatorname{grad} u$. By Lemma 2 we have

$$
\|u\|_{F^{*}} \leq C\left\|T^{*} u\right\|_{E^{*}} \quad \forall u \in F^{*},
$$

and therefore $T$ is surjective from $E$ onto $F$. Estimate (4.1) follows from the open mapping principle or one could argue directly using (4.2) and Hahn-Banach as in the proof of Proposition 1.

Proof of Lemma 2. Assume

$$
\begin{gathered}
u \in L_{\#}^{2}, \\
\partial_{x} u=F_{1}+h_{1}, \partial_{y} u=F_{2}+h_{2}
\end{gathered}
$$

and

$$
\left\|F_{1}\right\|_{L^{1}}+\left\|F_{2}\right\|_{L^{1}}+\left\|h_{1}\right\|_{H^{-1}}+\left\|h_{2}\right\|_{H^{-1}} \leq 1
$$


We have to prove that

$$
\|u\|_{L^{2}} \leq C
$$

The main ingredient is

Lemma 3. Under assumptions (4.4)-(4.6) we have

$$
\sum_{n_{1}, n_{2} \in \mathbb{Z}} \frac{n_{1}^{2} n_{2}^{2}}{\left(n_{1}^{2}+n_{2}^{2}\right)^{2}}\left|\hat{u}\left(n_{1}, n_{2}\right)\right|^{2} \leq C\left(\|u\|_{L^{2}}+1\right) .
$$

Assuming Lemma 3 we may now complete the proof of Lemma 2. Define

$$
u^{\prime}\left(x^{\prime}, y^{\prime}\right)=u\left(x^{\prime}+y^{\prime}, x^{\prime}-y^{\prime}\right)=\sum_{n_{1}, n_{2}} \hat{u}\left(n_{1}, n_{2}\right) e^{i\left[\left(n_{1}+n_{2}\right) x^{\prime}+\left(n_{1}-n_{2}\right) y^{\prime}\right]}
$$

so that

$$
\widehat{u^{\prime}}\left(n_{1}+n_{2}, n_{1}-n_{2}\right)=\hat{u}\left(n_{1}, n_{2}\right)
$$

and

$$
\begin{aligned}
\partial_{x^{\prime}} u^{\prime}\left(x^{\prime}, y^{\prime}\right) & =\partial_{x} u\left(x^{\prime}+y^{\prime}, x^{\prime}-y^{\prime}\right)+\partial_{y} u\left(x^{\prime}+y^{\prime}, x^{\prime}-y^{\prime}\right) \\
& =\left(F_{1}+F_{2}\right)\left(x^{\prime}+y^{\prime}, x^{\prime}-y^{\prime}\right)+\left(h_{1}+h_{2}\right)\left(x^{\prime}+y^{\prime}, x^{\prime}-y^{\prime}\right) \\
& \in L^{1}+H^{-1}
\end{aligned}
$$

and similarly for $\partial_{y^{\prime}} u^{\prime}$.

From (4.8) and (4.10) we obtain

$$
\begin{aligned}
& \sum_{n_{1}, n_{2}} \frac{\left(n_{1}+n_{2}\right)^{2}\left(n_{1}-n_{2}\right)^{2}}{4\left(n_{1}^{2}+n_{2}^{2}\right)^{2}}\left|\hat{u}\left(n_{1}, n_{2}\right)\right|^{2}=\sum_{n_{1}^{\prime}, n_{2}^{\prime}} \frac{\left(n_{1}^{\prime}\right)^{2}\left(n_{2}^{\prime}\right)^{2}}{\left(\left(n_{1}^{\prime}\right)^{2}+\left(n_{2}^{\prime}\right)^{2}\right)^{2}}\left|\widehat{u^{\prime}}\left(n_{1}^{\prime}, n_{2}^{\prime}\right)\right|^{2} \\
& \quad \leq C\left(\left\|u^{\prime}\right\|_{L^{2}}+1\right)=C\left(\|u\|_{L^{2}}+1\right) .
\end{aligned}
$$

Addition of (4.8) and (4.11) implies that

$$
\|u\|_{L^{2}}^{2}=\sum_{n_{1}, n_{2}}\left|\hat{u}\left(n_{1}, n_{2}\right)\right|^{2} \leq C\left(\|u\|_{L^{2}}+1\right)
$$

and the desired estimate (4.7) follows.

We now turn to the

Proof of Lemma 3. We have

$$
\begin{aligned}
& \sum_{n \neq 0} \frac{n_{1}^{2} n_{2}^{2}}{\left(n_{1}^{2}+n_{2}^{2}\right)^{2}}|\hat{u}(n)|^{2}=\frac{1}{i} n \sum \frac{n_{1} n_{2}^{2}}{\left(n_{1}^{2}+n_{2}^{2}\right)^{2}} \widehat{\partial_{x} u}(n) \hat{u}(-n) \\
& \quad \stackrel{\text { by }}{=}=\frac{(4.5)}{i} \sum \frac{n_{1} n_{2}^{2}}{\left(n_{1}^{2}+n_{2}^{2}\right)^{2}} \hat{F}_{1}(n) \hat{u}(-n)+\frac{1}{i} \sum \frac{n_{1} n_{2}^{2}}{\left(n_{1}^{2}+n_{2}^{2}\right)^{2}} \hat{h}_{1}(n) \hat{u}(-n) \\
& \quad=(4.12)+(4.13) .
\end{aligned}
$$

Estimate

$$
|(4.13)| \leq \sum_{n_{1}, n_{2}} \frac{\left|\hat{h}_{1}(n)\right|}{\sqrt{n_{1}^{2}+n_{2}^{2}}}|\hat{u}(-n)| \leq\left\|h_{1}\right\|_{H^{-1}}\|u\|_{L^{2}} .
$$


Write

$$
\begin{aligned}
(4.12) & =\sum \frac{n_{1} n_{2}}{\left(n_{1}^{2}+n_{2}^{2}\right)^{2}} \hat{F}_{1}(n) \widehat{\partial_{y} u}(-n) \\
& =\sum \frac{n_{1} n_{2}}{\left(n_{1}^{2}+n_{2}^{2}\right)^{2}} \hat{F}_{1}(n) \hat{F}_{2}(-n)+\sum \frac{n_{1} n_{2}}{\left(n_{1}^{2}+n_{2}^{2}\right)^{2}} \hat{F}_{1}(n) \hat{h}_{2}(-n) \\
& =(4.15)+(4.16) .
\end{aligned}
$$

Estimate

$$
\begin{aligned}
|(4.16)| & \leq \sum \frac{\left|n_{1}\right|\left|n_{2}\right|}{\left(n_{1}^{2}+n_{2}^{2}\right)^{2}}\left(\left|\widehat{\partial_{x} u}(n)\right|+\left|\hat{h}_{1}(n)\right|\right)\left|\hat{h}_{2}(-n)\right| \\
& \leq \sum \frac{n_{1}^{2}\left|n_{2}\right|}{\left(n_{1}^{2}+n_{2}^{2}\right)^{2}}|\hat{u}(n)|\left|\hat{h}_{2}(-n)\right|+\sum \frac{\left|\hat{h}_{1}(n)\right|}{\sqrt{n_{1}^{2}+n_{2}^{2}}} \frac{\left|\hat{h}_{2}(-n)\right|}{\sqrt{n_{1}^{2}+n_{2}^{2}}} \\
& \leq\|f\|_{L^{2}}\left\|h_{2}\right\|_{H^{-1}}+\left\|h_{1}\right\|_{H^{-1}}\left\|h_{2}\right\|_{H^{-1}} .
\end{aligned}
$$

Estimation of (4.15). This is the key point. Since $\left\|F_{1}\right\|_{L^{1}} \leq 1,\left\|F_{2}\right\|_{L^{1}} \leq 1$, it suffices (by convexity) to replace $\widehat{F}_{i}(n)$ by

$$
\widehat{F_{1}}(n)=e^{i n \cdot a}, \quad \widehat{F_{2}}(n)=e^{i n \cdot b}
$$

for some $a, b \in \mathbb{T}^{2}$ (this amounts to replacing $F_{1}, F_{2}$ by the Dirac measures $\delta_{a}, \delta_{b}$, respectively).

Thus we obtain

$$
\begin{aligned}
\sum_{n_{1}, n_{2} \in \mathbb{Z}} \frac{n_{1} n_{2}}{\left(n_{1}^{2}+n_{2}^{2}\right)^{2}} \hat{F}_{1}(n) \hat{F}_{2}(-n) & =\sum \frac{n_{1} n_{2}}{\left(n_{1}^{2}+n_{2}^{2}\right)^{2}} e^{i\left[n_{1}\left(a_{1}-b_{1}\right)+n_{2}\left(a_{2}-b_{2}\right)\right]} \\
& =-\sum \frac{n_{1} n_{2}}{\left(n_{1}^{2}+n_{2}\right)^{2}} \sin n_{1}\left(a_{1}-b_{1}\right) \sin n_{2}\left(a_{2}-b_{2}\right)
\end{aligned}
$$

by parity considerations.

Claim. For all $\theta_{1}, \theta_{2} \in \mathbb{T}$

$$
\left|\sum_{n_{1}, n_{2}} \frac{n_{1} n_{2}}{\left(n_{1}^{2}+n_{2}^{2}\right)^{2}} \sin n_{1} \theta_{1} \sin n_{2} \theta_{2}\right| \leq C .
$$

From the claim, we conclude that $|(4.15)|,|(4.19)| \leq C$ and, recalling also (4.14), (4.17), inequality (4.8) follows.

Proof of the Claim. Splitting $\mathbb{Z}$ in dyadic intervals, we obtain

$$
\sum_{k_{1}, k_{2} \geq 0}\left|\sum_{n_{1} \sim 2^{k_{1}, n_{2} \sim 2^{k_{2}}}} \frac{n_{1} n_{2}}{\left(n_{1}^{2}+n_{2}^{2}\right)^{2}} \sin n_{1} \theta_{1} \sin n_{2} \theta_{2}\right| .
$$

Recall the inequality

$$
\left|\sum_{n \in I} \sin n \theta\right| \lesssim 4^{k}|\theta| \wedge \frac{1}{|\theta|}
$$

if $\theta \in \mathbb{T}$ and $I \subset\left[2^{k-1}, 2^{k}\right]$ is an interval (where $\wedge$ denotes min). 
From (4.22), assuming $k_{1} \geq k_{2}$, we have

$$
\begin{aligned}
& \left|\sum_{n_{1} \sim 2^{k_{1}, n_{2} \sim 2^{k_{2}}}} \frac{n_{1} n_{2}}{\left(n_{1}^{2}+n_{2}^{2}\right)^{2}} \sin n_{1} \theta_{1} \sin n_{2} \theta_{2}\right| \leq \\
& \left(4^{k_{1}}\left|\theta_{1}\right| \wedge \frac{1}{\left|\theta_{1}\right|}\right)\left(4^{k_{2}}\left|\theta_{2}\right| \wedge \frac{1}{\left|\theta_{2}\right|}\right)\left\|\left\{\frac{n_{1} n_{2}}{\left(n_{1}^{2}+n_{2}^{2}\right)^{2}}\right\}\right\|_{\ell^{\infty}\left(n_{1} \sim 2^{k_{1}}\right) \hat{\otimes} \ell^{\infty}\left(n_{2} \sim 2^{k_{2}}\right)}
\end{aligned}
$$

where $\ell^{\infty}(I) \hat{\otimes} \ell^{\infty}(J)$ denotes the usual projective tensor product. Thus the last factor in (4.23) may be bounded by

$$
\left\|\partial_{n_{1} n_{2}}^{2} \frac{n_{1} n_{2}}{\left(n_{1}^{2}+n_{2}^{2}\right)^{2}}\right\|_{\ell^{1}\left(n_{1} \sim 2^{k_{1}}, n_{2} \sim 2^{k_{2}}\right)} \leq C\left\|\frac{1}{\left(n_{1}^{2}+n_{2}^{2}\right)^{2}}\right\|_{\ell^{1}\left(n_{1} \sim 2^{k_{1}}, n_{2} \sim 2^{k_{2}}\right)} \leq C \frac{2^{k_{2}}}{8^{k_{1}}} .
$$

Substitution of (4.23), (4.24) in (4.21) gives the bound

$$
\begin{aligned}
(4.20),(4.21) & \leq C \sum_{k_{1} \geq k_{2} \geq 0} 4^{k_{2}-k_{1}}\left(2^{k_{1}}\left|\theta_{1}\right| \wedge \frac{1}{2^{k_{1}}\left|\theta_{1}\right|}\right)\left(2^{k_{2}}\left|\theta_{2}\right| \wedge \frac{1}{2^{k_{2}}\left|\theta_{2}\right|}\right) \\
& \lesssim C \prod_{i=1}^{2}\left[\sum_{k \in \mathbb{Z}_{+}}\left(2^{k}\left|\theta_{i}\right| \wedge \frac{1}{2^{k}\left|\theta_{i}\right|}\right)\right] \leq C .
\end{aligned}
$$

This completes the proof of the Claim and of Theorem $1^{\prime}$ for $d=2$.

\section{Proof of Theorem 1 When $d=2$ (explicit Construction)}

Our aim is to construct $Y \in L^{\infty} \cap H^{1}$ such that

$$
\operatorname{div} Y=f \in L_{\#}^{2}\left(\mathbb{T}^{2}\right) \text {. }
$$

Write

$$
\mathbb{Z}^{2}=\bigcup_{j \geq 0}\left(\Lambda_{j}^{1} \cup \Lambda_{j}^{2}\right)
$$

where

$$
\begin{aligned}
& \Lambda_{j}^{1}=\left[2^{j-1}<\left|n_{1}\right| \leq 2^{j} ;\left|n_{2}\right| \leq 2^{j}\right] \\
& \Lambda_{j}^{2}=\left[2^{j}<\left|n_{2}\right| \leq 2^{j+1} ;\left|n_{1}\right| \leq 2^{j}\right] .
\end{aligned}
$$

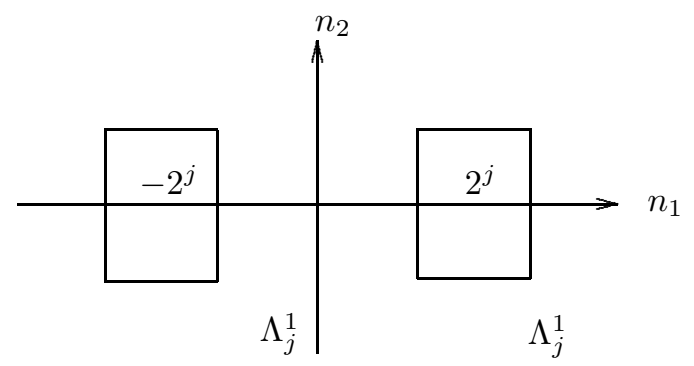

Let

$$
\Lambda^{\alpha}=\bigcup_{j} \Lambda_{j}^{\alpha} \quad(\alpha=1,2)
$$

Decompose

$$
f=f^{1}+f^{2} \text { where } f^{\alpha}=P_{\Lambda^{\alpha}} f \equiv \sum_{n \in \Lambda^{\alpha}} \hat{f}(n) e^{i n \cdot x}
$$


Claim. Let $\delta>0$ be small enough and $\|f\|_{2} \leq \delta$. Then there are $Y_{1}, Y_{2}$ such that

$$
\left\|Y_{\alpha}\right\|_{L^{\infty} \cap H^{1}} \leq 1
$$

and

$$
\left\|\partial_{\alpha} Y_{\alpha}-f^{\alpha}\right\|_{2} \leq \delta^{4 / 3} \quad(\alpha=1,2)
$$

Thus if $\|f\|_{2}=\delta$, then

$$
\left\|f-\partial_{1} Y_{1}-\partial_{2} Y_{2}\right\|_{2} \leq \delta^{1 / 3}\|f\|_{2}
$$

and iteration of this gives (5.1).

The construction of $Y_{1}, Y_{2}$ is explicit but nonlinear (see Proposition 2).

Take $\alpha=1$ and denote $f^{1}$ by $f, \Lambda_{j}^{1}$ by $\Lambda_{j}$.

Define

$$
\begin{aligned}
f_{j} & =P_{\Lambda_{j}} f, \\
c_{j} & =\left\|f_{j}\right\|_{2}, \\
F_{j} & =D_{x_{1}}^{-1} f_{j} \equiv \sum \frac{1}{n_{1}} \hat{f}_{j}(n) e^{i n \cdot x} .
\end{aligned}
$$

Hence

$$
\begin{gathered}
\left(\sum c_{j}^{2}\right)^{1 / 2}=\|f\|_{2}, \\
\left\|F_{j}\right\|_{\infty} \leq \sum_{n \in \Lambda_{j}} \frac{1}{\left|n_{1}\right|}|\hat{f}(n)| \lesssim 2^{-j}\left|\Lambda_{j}\right|^{1 / 2}\left\|f_{j}\right\|_{2} \lesssim c_{j} .
\end{gathered}
$$

Fix $\varepsilon>0$ a small constant and partition

$$
\Lambda_{j}=\bigcup_{r<\frac{1}{\varepsilon}+1} \Lambda_{j, r}
$$

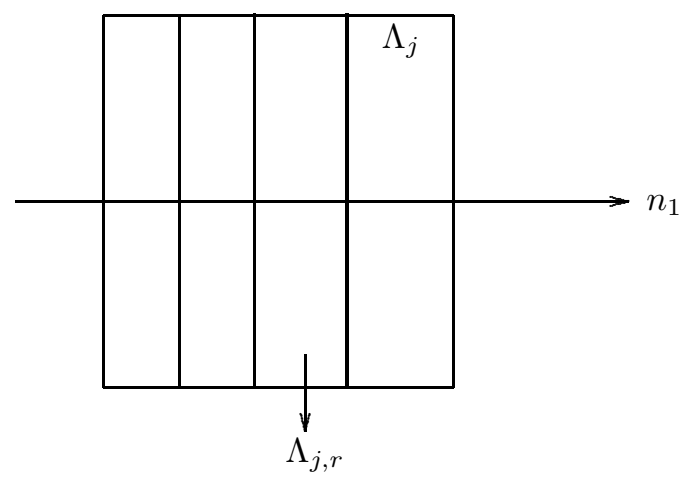

in stripes $\Lambda_{j, r}$ such that

$$
\left|\operatorname{Proj}_{n_{1}} \Lambda_{j, r}\right| \sim \varepsilon 2^{j}
$$

Define first

$$
\tilde{F}_{j}(x)=\sum_{r}\left|\sum_{n \in \Lambda_{j, r}} \frac{1}{n_{1}} \hat{f}_{j}(n) e^{i n \cdot x}\right| .
$$

Thus

$$
\left|F_{j}(x)\right| \leq\left|\tilde{F}_{j}(x)\right| \lesssim c_{j} .
$$


From Cauchy-Schwarz

$$
\left\|\tilde{F}_{j}\right\|_{2} \leq \varepsilon^{-1 / 2}\left\|F_{j}\right\|_{2} \lesssim \varepsilon^{-1 / 2} 2^{-j} c_{j} .
$$

Observe that if $\operatorname{Proj}_{n_{1}} \Lambda_{j, r}=\left[a_{r}, b_{r}\right], b_{r}-a_{r} \sim \varepsilon 2^{j}$, then

$$
\left|\partial_{1} \tilde{F}_{j}\right| \leq \sum_{r}\left|\sum_{n \in \Lambda_{j, r}} \frac{n_{1}-a_{r}}{n_{1}} \hat{f}_{j}(n) e^{i n \cdot x}\right|
$$

where

$$
\left|\frac{n_{1}-a_{r}}{n_{1}}\right|<\varepsilon
$$

Therefore

$$
\left\|\partial_{1} \tilde{F}_{j}\right\|_{2} \lesssim \sum_{r} \varepsilon\left\|P_{\Lambda_{j, r}} f\right\|_{2} \lesssim \varepsilon^{1 / 2}\left\|P_{\Lambda_{j}} f\right\|_{2}=\varepsilon^{1 / 2} c_{j}
$$

(this is the purpose of the construction of $\tilde{F}_{j}$ ).

We also need to make an appropriate localization of the Fourier transform of $\tilde{F}_{j}$. Denote

$$
K_{N}(y)=\sum_{|n|<N} \frac{N-|n|}{N} e^{i n y},
$$

the usual Féjer kernel on $\mathbb{T}$. It is easy to see that if

$$
P(y)=\sum_{|n|<N} \hat{P}(n) e^{i n y}
$$

is a trigonometric polynomial, then

$$
|P| \leq 3\left(|P| * K_{N}\right) .
$$

Using this fact in the variables $x_{1}, x_{2}$, we see that

$$
\left|F_{j}\right| \leq \tilde{F}_{j} \leq G_{j}
$$

denoting

$$
G_{j}=9 \tilde{F}_{j} *\left(K_{N_{1}} \otimes K_{N_{2}}\right)
$$

where each $\Delta_{j, r}$ is an $N_{1} \times N_{2}$ rectangle, $N_{1} \sim \varepsilon 2^{j}, N_{2} \sim 2^{j}$.

Thus, by construction

$$
\operatorname{supp} \hat{G}_{j} \subset\left[-N_{1}, N_{1}\right] \times\left[-N_{2}, N_{2}\right] \subset\left[|n| \leq 2^{j}\right]
$$

and inequalities (5.7), (5.8), (5.9) remain preserved.

Therefore,

$$
\begin{aligned}
\left\|G_{j}\right\|_{\infty} & \leq 9\left\|\tilde{F}_{j}\right\|_{\infty} \lesssim c_{j} \quad(0<\delta<1), \\
\left\|G_{j}\right\|_{2} & \lesssim \varepsilon^{-1 / 2} 2^{-j} c_{j}, \\
\left\|\partial_{1} G_{j}\right\|_{2} & \lesssim \varepsilon^{1 / 2} c_{j}, \\
\left\|\nabla G_{j}\right\|_{2} & \lesssim \varepsilon^{-1 / 2} c_{j} .
\end{aligned}
$$

Assume that $\left\{f_{j} \mid j \leq K\right\}$ is a finite sequence (which is no restriction). 
Define

$$
\begin{aligned}
Y_{1}= & F_{K}+F_{K-1}\left(1-G_{K}\right) \\
& +F_{K-2}\left(1-G_{K-1}\right)\left(1-G_{K}\right)+\cdots \\
= & \sum_{j \leq K} F_{j} \prod_{k>j}\left(1-G_{k}\right) .
\end{aligned}
$$

Thus from (5.11)

$$
\begin{aligned}
\left|Y_{1}\right| \leq\left|F_{K}\right| & +\left(1-\left|F_{K}\right|\right)\left|F_{K-1}\right| \\
& +\left(1-\left|F_{K}\right|\right)\left(1-\left|F_{K-1}\right|\right)\left|F_{K-2}\right|+\cdots \leq 1 .
\end{aligned}
$$

One may also rewrite (5.18) as

$$
Y_{1}=\sum F_{j}-\sum G_{j} H_{j}
$$

with

$$
\begin{aligned}
H_{j}= & F_{j-1}+F_{j-2}\left(1-G_{j-1}\right) \\
& +F_{j-3}\left(1-G_{j-2}\right)\left(1-G_{j-1}\right)+\cdots \\
= & \sum_{k<j} F_{k} \prod_{k<k^{\prime}<j}\left(1-G_{k^{\prime}}\right) .
\end{aligned}
$$

Clearly

$$
\left|H_{j}\right|<1
$$

By construction

$$
\partial_{1} Y_{1}=\sum f_{j}-\sum \partial_{1}\left(G_{j} H_{j}\right)
$$

Next, we estimate the second term in (5.21) that will appear as an error term.

Observe that since $\operatorname{supp} \hat{F}_{j} \subset\left[|n| \sim 2^{j}\right]$ and (5.13), also

$$
\operatorname{supp} \hat{H}_{j} \subset\left[|n| \lesssim 2^{j}\right] \text {. }
$$

Denote $P_{k}$ Fourier projection operators on $\left[|n| \sim 2^{k}\right]$ such that $I d=\sum_{k \geq 0} P_{k}$.

From the preceding, we may thus ensure that

$$
G_{j} H_{j}=\sum_{k \leq j} P_{k}\left(G_{j} H_{j}\right) .
$$

Estimate then

$$
\left\|\sum_{j} \partial_{1}\left(G_{j} H_{j}\right)\right\|_{2} \leq \sum_{s \geq 0}\left(\sum_{j}\left\|\partial_{1} P_{j-s}\left(G_{j} H_{j}\right)\right\|_{2}^{2}\right)^{1 / 2}
$$

(since for fixed $s$, the $P_{j-s}$ have disjoint ranges).

Returning to the parameter $0<\varepsilon<1$ introduced earlier, write

$$
\varepsilon=2^{-s_{*}}\left(s_{*}>0\right)
$$

and estimate (5.24) in the ranges

$$
\begin{aligned}
& s>s_{*} \\
& 0 \leq s \leq s_{*} .
\end{aligned}
$$


Contribution of (5.26). Since $\left|H_{j}\right| \leq 1$ and (5.15),

$$
\begin{aligned}
\left\|\partial_{1} P_{j-s}\left(G_{j} H_{j}\right)\right\|_{2} & \lesssim 2^{j-s}\left\|G_{j} H_{j}\right\|_{2} \\
& \leq 2^{j-s}\left\|G_{j}\right\|_{2} \leq \varepsilon^{-1 / 2} 2^{-s} c_{j} .
\end{aligned}
$$

Substitution in (5.24) gives the contribution

$$
\sum_{s \geq s_{*}} 2^{-s} \varepsilon^{-1 / 2}\left(\sum c_{j}^{2}\right)^{1 / 2}<2^{-s_{*}} \varepsilon^{-1 / 2}\|f\|_{2}<\varepsilon^{1 / 2}\|f\|_{2} .
$$

Contribution of (5.27). Estimate now

$$
\begin{aligned}
\left\|\partial_{1} P_{j-s}\left(G_{j} H_{j}\right)\right\|_{2} \leq\left\|\partial_{1}\left(G_{j} H_{j}\right)\right\|_{2} & \leq\left\|\partial_{1} G_{j}\right\|_{2}+\left\|G_{j} \partial_{1} H_{j}\right\|_{2} \\
& \leq \varepsilon^{1 / 2} c_{j}+\left\|G_{j} \partial_{1} H_{j}\right\|_{2}
\end{aligned}
$$

using (5.16).

Recalling definition (5.20) of $H_{j}$, one easily verifies that

$$
\left|\nabla H_{j}\right| \leq \sum_{k<j}\left(\left|\nabla F_{k}\right|+\left|\nabla G_{k}\right|\right) .
$$

Hence

$$
\left\|\nabla H_{j}\right\|_{\infty} \leq \sum_{k<j} 2^{k} c_{k}
$$

and from $(5.15)$

$$
\left\|G_{j} \partial_{1} H_{j}\right\|_{2} \leq \varepsilon^{-1 / 2} c_{j}\left(\sum_{k<j} 2^{-(j-k)} c_{k}\right) .
$$

Substitution of (5.30), (5.33) in (5.24) gives the following bound on the contribution of (5.27):

$$
\begin{gathered}
s_{*} \varepsilon^{1 / 2}\left(\sum c_{j}^{2}\right)^{1 / 2}+s_{*} \varepsilon^{-1 / 2}\left[\sum_{j} c_{j}^{2}\left(\sum_{k<j} 2^{-(j-k)} c_{k}\right)^{2}\right]^{1 / 2} \\
\leq\left(\log \frac{1}{\varepsilon}\right) \varepsilon^{1 / 2}\|f\|_{2}+\left(\log \frac{1}{\varepsilon}\right) \varepsilon^{-1 / 2}\|f\|_{2}^{2} .
\end{gathered}
$$

Consequently, from (5.21), (5.29), (5.34),

$$
\left\|f-\partial_{1} Y_{1}\right\|_{2}=\left\|\sum_{j} \partial_{1}\left(G_{j} H_{j}\right)\right\|_{2} \leq \log \frac{1}{\varepsilon}\left(\varepsilon^{1 / 2}\|f\|_{2}+\varepsilon^{-1 / 2}\|f\|_{2}^{2}\right) .
$$

Under the assumption $\|f\|_{2} \leq \delta$, letting $\varepsilon=\delta$ in (5.35), we obtain thus

$$
\left\|f-\partial_{1} Y_{1}\right\|_{2} \leq \delta^{\frac{3}{2}-} \leq \delta^{\frac{4}{3}}
$$

which is (5.3).

It remains to estimate $\left\|Y_{1}\right\|_{H^{1}}=\left\|\nabla Y_{1}\right\|_{2}$.

By (5.19)

$$
\left\|\nabla Y_{1}\right\|_{2} \leq\left\|\sum_{j} \nabla F_{j}\right\|_{2}+\left\|\sum \nabla\left(G_{j} H_{j}\right)\right\|_{2}
$$


From the definition of $F_{j}$ and since $\operatorname{supp} \hat{F}_{j} \subset \Lambda_{j}^{1}$, it follows that

$$
\left\|\sum_{j} \nabla F_{j}\right\|_{2} \sim\left(\sum\left\|f_{j}\right\|_{2}^{2}\right)^{1 / 2}=\|f\|_{2} .
$$

Estimate the second term in (5.37) as in (5.24),

$$
\left\|\sum_{j} \nabla\left(G_{j} H_{j}\right)\right\|_{2} \leq \sum_{s \geq 0}\left(\sum_{j}\left\|\nabla P_{j-s}\left(G_{j} H_{j}\right)\right\|_{2}^{2}\right)^{1 / 2}
$$

and

$$
\left\|\nabla P_{j-s}\left(G_{j} H_{j}\right)\right\|_{2} \lesssim 2^{j-s}\left\|G_{j} H_{j}\right\|_{2} \leq \varepsilon^{-1 / 2} 2^{-s} c_{j} .
$$

Thus

$$
(5.39) \leq \varepsilon^{-1 / 2} \sum_{s \geq 0} 2^{-s}\left(\sum_{j} c_{j}^{2}\right)^{1 / 2} \leq \varepsilon^{-1 / 2}\|f\|_{2}
$$

and

$$
\left\|\nabla Y_{1}\right\|_{2} \leq \delta^{-1 / 2}\|f\|_{2} \leq \delta^{1 / 2} .
$$

Since $\left\|Y_{1}\right\|_{\infty} \lesssim 1$, this establishes (5.2).

This proves the Claim and completes the proof of Theorem 1 for $d=2$.

\section{Proof of Theorem 1 When $d>2$ (explicit construction)}

Let $f \in L_{\#}^{d}\left(\mathbb{T}^{d}\right)$. Our aim is to construct a solution $Y$ of $\operatorname{div} Y=f$ satisfying

$$
\begin{aligned}
\|Y\|_{\infty} & \leq C\|f\|_{d}, \\
\|\nabla Y\|_{d} & \leq C\|f\|_{d} .
\end{aligned}
$$

We do this by standard modification of the previous $L^{2}$-argument with the Littlewood-Paley square function theory as main additional ingredient. Consider again a partition

$$
\mathbb{Z}^{d}=\bigcup_{j \geq 0}\left(\Lambda_{j}^{1} \cup \cdots \cup \Lambda_{j}^{d}\right)
$$

of disjoint $d$-rectangles $\Lambda_{j}^{\alpha}$ of side length $\sim 2^{j}$.

We formulate the analogue of the Claim with $Y_{\alpha}$ satisfying bounds (6.1), (6.2). Letting $\alpha=1, f=f^{1}$, define again

$$
F_{j}=D_{x_{1}}^{-1} f_{j}
$$

satisfying

$$
\left\|F_{j}\right\|_{\infty} \lesssim\left(2^{j / d}\right)^{d}\left\|F_{j}\right\|_{d}=2^{j}\left\|D_{x_{1}}^{-1} f_{j}\right\|_{d} \sim\left\|f_{j}\right\|_{d} \equiv c_{j} .
$$


Define $\tilde{F}_{j}$ and $G_{j}$ as in (5.6), (5.12). Thus (5.11), (5.13) hold. Also

$$
\begin{aligned}
\left\|G_{j}\right\|_{\infty} & \lesssim\left\|\tilde{F}_{j}\right\|_{\infty} \leq \varepsilon^{-1 / d^{\prime}}\left(\sum_{r<\frac{1}{\varepsilon}}\left\|\sum_{n \in \Lambda_{j, r}} \frac{1}{n_{1}} \hat{f}_{j}(n) e^{i n x}\right\|_{\infty}^{d}\right)^{1 / d} \\
& \leq \varepsilon^{-1 / d^{\prime}}\left(\sum_{r<\frac{1}{\varepsilon}}\left(2^{j \frac{d-1}{d}}\left(\varepsilon 2^{j}\right)^{\frac{1}{d}}\left\|\sum_{n \in \Lambda_{j, r}} \frac{1}{n_{1}} \hat{f}_{j}(n) e^{i n \cdot x}\right\|_{d}\right)^{d}\right)^{1 / d} \\
& \lesssim \varepsilon^{-1 / d^{\prime}+1 / d}\left(\sum_{r<\frac{1}{\varepsilon}}\left\|\sum_{n \in \Lambda_{j, r}} \hat{f}_{j}(n) e^{i n x}\right\|_{d}^{d}\right)^{\frac{1}{d}} \\
& \lesssim \varepsilon^{\frac{2}{d}-1}\left\|f_{j}\right\|_{d}=\varepsilon^{\frac{2}{d}-1} c_{j} \leq \varepsilon^{\frac{2}{d}-1} \delta .
\end{aligned}
$$

(We assume that $\delta$ is small enough compared with $\varepsilon$ to ensure, in particular, that $\varepsilon^{\frac{2}{d}-1} \delta \ll 1$.)

Repeat the construction from Section 5. In place of estimate (5.24) we now have

$$
\left.\left\|\sum_{j} \partial_{1}\left(G_{j} H_{j}\right)\right\|_{d} \leq \sum_{s \geq 0} \| \sum_{j}\left|\partial_{1} P_{j-s}\left(G_{j} H_{j}\right)\right|^{2}\right)^{1 / 2} \|_{d}
$$

and distinguish between the cases (5.26), (5.27).

Contribution of (5.26). Estimate

$$
\begin{gathered}
\left\|\left(\sum_{j}\left|\nabla P_{j-s}\left(G_{j} H_{j}\right)\right|^{2}\right)^{1 / 2}\right\|_{d} \\
\lesssim\left\|\left(\sum_{j} 4^{j-s}\left|P_{j-s}\left(G_{j} H_{j}\right)\right|^{2}\right)^{1 / 2}\right\|_{d} \\
\lesssim 2^{-s}\left\|\left(\sum_{j} 4^{j}\left|G_{j} H_{j}\right|^{2}\right)^{1 / 2}\right\|_{d} \\
\lesssim 2^{-s}\left\|\left(\sum_{j} 4^{j}\left(\tilde{F}_{j} * K_{j}\right)^{2}\right)^{1 / 2}\right\|_{d}
\end{gathered}
$$

where $K_{j}$ is a product of Féjer kernels

$$
K_{N_{1}} \otimes K_{N_{2}} \otimes \cdots \otimes K_{N_{d}}, \quad N_{1} \sim \varepsilon 2^{j}, \quad \text { and } N_{2}, \ldots, N_{d} \sim 2^{j} .
$$

Again from standard square function inequalities

$$
(6.7) \lesssim 2^{-s}\left\|\left(\sum_{j} 4^{j}\left(\tilde{F}_{j}\right)^{2}\right)^{1 / 2}\right\|_{d}
$$

Recalling the definition of $\tilde{F}_{j}$, estimate

$$
\left(\tilde{F}_{j}\right)^{2} \leq \varepsilon^{-1} \sum_{r \leq \varepsilon^{-1}}\left|\sum_{n \in \Lambda_{j, r}^{1}} \frac{1}{n_{1}} \hat{f}(n) e^{i n x}\right|^{2} .
$$


Substituting in (6.8), this gives

$$
\begin{aligned}
& \varepsilon^{-1 / 2} 2^{-s}\left\|\left(\sum_{j} \sum_{r<\varepsilon^{-1}}\left|\sum_{n \in \Lambda_{j, r}^{1}} \frac{2^{j}}{n_{1}} \hat{f}(n) e^{i n x}\right|^{2}\right)^{1 / 2}\right\|_{d} \\
& \lesssim \varepsilon^{-1 / 2} 2^{-s}\left\|\left(\sum_{j} \sum_{r<\varepsilon^{-1}}\left|\sum_{n \in \Lambda_{j, r}^{1}} \hat{f}(n) e^{i n x}\right|^{2}\right)^{1 / 2}\right\|_{d}
\end{aligned}
$$

We use here the fact that $\left|n_{1}\right| \sim|n| \sim 2^{j}$ for $n \in \Lambda_{j}^{1}$.

Recall also the definition of $\Lambda_{j, r}$ obtained by partitioning the $n_{1}$-variable in intervals of size $\varepsilon 2^{j}$.

At this stage, we use the following (1-variable) inequality due to Rubio de Francia [19], which generalizes the Littlewood-Paley inequality to arbitrary intervals.

Proposition 3. Let $\left\{I_{\alpha}\right\}$ be disjoint intervals in $\mathbb{Z}$ and

$$
P_{I} f=\sum_{n \in I} \hat{f}(n) e^{i n x}
$$

the corresponding Fourier projection.

Then, for $2 \leq d<\infty$, there is the (one-sided) inequality

$$
\left\|\left(\sum\left|P_{I_{\alpha}} f\right|^{2}\right)^{1 / 2}\right\|_{d} \leq C\|f\|_{d}
$$

Since $\left\{\operatorname{Proj}_{n_{1}} \Lambda_{j r}^{1}\right\}$ are disjoint intervals in $\mathbb{Z}$, application of (6.11) in the $x_{1^{-}}$ variable implies that

$$
(6.6) \lesssim \varepsilon^{-1 / 2} 2^{-s}\|f\|_{d}
$$

Summation of (6.12) for $s \geq s_{*}$ gives then

$$
\text { (5.26)-contribution } \leq \varepsilon^{1 / 2}\|f\|_{d} .
$$

Remark 8. We used the general Proposition 3 for convenience; the present case could in fact be treated by more elementary means.

Contribution of (5.27). Estimate

$$
\begin{aligned}
& \left\|\left(\sum_{j}\left|\partial_{1} P_{j-s}\left(G_{j} H_{j}\right)\right|^{2}\right)^{1 / 2}\right\|_{d} \lesssim\left\|\left(\sum_{j}\left|\partial_{1}\left(G_{j} H_{j}\right)\right|^{2}\right)^{1 / 2}\right\|_{d} \\
& \leq\left\|\left(\sum_{j}\left|\partial_{1} G_{j}\right|^{2}\right)^{1 / 2}\right\|_{d}+\left\|\left(\sum_{j}\left|G_{j}\left(\partial_{1} H_{j}\right)\right|^{2}\right)^{1 / 2}\right\|_{d}=(6.14)+(6.15) .
\end{aligned}
$$

Estimate (6.14) by

$$
\left\|\left(\sum_{j}\left|\partial_{1} \tilde{F}_{j}\right|^{2}\right)^{1 / 2}\right\|_{d}
$$


We have that

$$
\begin{aligned}
\left|\partial_{1} \tilde{F}_{j}\right| & \leq \sum_{r<\varepsilon^{-1}}\left|\sum_{n \in \Lambda_{j, r}^{1}} \frac{n_{1}-a_{j, r}}{n_{1}} \hat{f}(n) e^{i n x}\right| \\
& \leq \varepsilon^{-1 / 2}\left(\sum_{r<\varepsilon^{-1}}\left|\sum_{n \in \Lambda_{j r}^{1}} \frac{n_{1}-a_{j, r}}{n_{1}} \hat{f}(n) e^{i n x}\right|^{2}\right)^{1 / 2}
\end{aligned}
$$

where $\operatorname{Proj}_{n_{1}} \Lambda_{j r}^{1}=\left[a_{j r}, b_{j r}\right], b_{j r}-a_{j r} \sim \varepsilon 2^{j}$. Thus $\left|\frac{n_{1}-a_{j, r}}{n_{1}}\right| \leq \varepsilon$.

We get therefore

$$
\begin{aligned}
(6.16) & \leq \varepsilon^{-1 / 2} \cdot \varepsilon\left\|\left(\sum_{j} \sum_{r<\varepsilon^{-1}}\left|\sum_{n \in \Lambda_{j r}^{1}} \hat{f}(n) e^{i n x}\right|^{2}\right)^{1 / 2}\right\|_{d} \\
& \lesssim \varepsilon^{1 / 2}\|f\|_{d} .
\end{aligned}
$$

To estimate (6.15), use again inequality (5.31), together with (6.4), (6.5). Thus

$$
\left\|\nabla H_{j}\right\|_{\infty} \leq \varepsilon^{\frac{2}{d}-1} \sum_{k<j} 2^{k} c_{k}<\varepsilon^{\frac{2}{d}-1} 2^{j}\|f\|_{d} .
$$

Hence

$$
\begin{aligned}
(6.15) & \leq \varepsilon^{\frac{2}{d}-1}\|f\|_{d}\left\|\left(\sum_{j} 4^{j} G_{j}^{2}\right)^{1 / 2}\right\|_{d} \\
& \leq \varepsilon^{\frac{2}{d}-1}\|f\|_{d}\left\|\left(\sum_{j}\left(2^{j} \tilde{F}_{j}\right)^{2}\right)^{1 / 2}\right\|_{d} \\
& \leq \varepsilon^{\frac{2}{d}-\frac{3}{2}}\|f\|_{d}^{2}
\end{aligned}
$$

applying again the (6.8)-bound using Proposition 3.

Thus the (5.27)-contribution is

$$
\leq \varepsilon^{1 / 2} \log \frac{1}{\varepsilon}\|f\|_{d}+\varepsilon^{\frac{2}{d}-\frac{3}{2}} \log \frac{1}{\varepsilon}\|f\|_{d}^{2} .
$$

Collecting estimates (6.13), (6.20), it follows that

$$
\begin{aligned}
\left\|f-\partial_{1} Y\right\|_{d} & =\left\|\sum_{j} \partial_{1}\left(G_{j} H_{j}\right)\right\|_{d} \\
& \leq \varepsilon^{1 / 2} \log \frac{1}{\varepsilon}\|f\|_{d}+\varepsilon^{\frac{2}{d}-\frac{3}{2}} \log \frac{1}{\varepsilon}\|f\|_{d}^{2}
\end{aligned}
$$

which is the analogue of (5.35). Assuming $\|f\|_{d}=\delta$, take $\varepsilon=\delta^{1 / 2}$ to obtain

$$
\left\|f-\partial_{1} Y\right\|_{d} \leq \delta^{1 / 5}\|f\|_{d} .
$$

It remains to estimate

$$
\|\nabla Y\|_{d} \leq\left\|\sum \nabla F_{j}\right\|_{d}+\left\|\sum \nabla\left(G_{j} H_{j}\right)\right\|_{d}=(6.23)+(6.24) .
$$

We have

$$
(6.23) \sim\left\|\left(\sum\left|\nabla F_{j}\right|^{2}\right)^{1 / 2}\right\|_{d} \sim\left\|\left(\sum\left|f_{j}\right|^{2}\right)^{1 / 2}\right\|_{d} \lesssim\|f\|_{d} .
$$


Estimate (6.24) as

$$
\left\|\sum_{s \geq 0}\left(\sum_{j}\left|\nabla P_{j-s}\left(G_{j} H_{j}\right)\right|^{2}\right)^{1 / 2}\right\|_{d} \lesssim \varepsilon^{-1 / 2}\|f\|_{d}
$$

using (6.7)-(6.12).

This completes the argument.

We conclude this section with a

Proof of Theorem $1^{\prime}$ when $d>2$. The argument is somewhat bizarre: one uses duality twice! First, from Theorem 1 we easily deduce the estimate on $\mathbb{T}^{d}$

$$
\left\|u-\int u\right\|_{L^{d /(d-1)}} \leq C(d)\|\operatorname{grad} u\|_{L^{1}+W^{-1, d /(d-1)}}, \forall u \in L^{d /(d-1)} .
$$

Next, we argue as in the beginning of Section 4. Observe that

$$
L^{1}+W^{-1, d /(d-1)} \subset \mathcal{M}+H^{-1}
$$

and that

$$
\|\cdots\|_{L^{1}+W^{-1, d /(d-1)}}=\|\cdots\|_{\mathcal{M}+W^{-1, d /(d-1)}} \text { on } L^{1}+W^{-1, d /(d-1)}
$$

(this may be easily seen using regularization by convolution).

Let $E=C^{0} \cap W^{1, d}, F=L_{\#}^{d}$ and consider the bounded operator $T: E \rightarrow F$ defined by $T Y=\operatorname{div} Y$. Clearly $T^{*}: F^{*} \rightarrow E^{*}=\mathcal{M}+W^{-d, d /(d-1)}$ is given by $T^{*} u=\operatorname{grad} u$. By (6.26) and (6.27) we obtain

$$
\|u\|_{F^{*}} \leq C\left\|T^{*} u\right\|_{E^{*}} \quad \forall u \in F^{*}
$$

and therefore $T$ is surjective from $E$ onto $F$. Applying the open mapping principle (or use Hahn-Banach as in the proof of Proposition 1), we see that for every $f \in F$ there is some $Y \in E$ satisfying $T Y=f$ and $\|Y\|_{E} \leq C\|f\|_{F}$.

Remark 9. Alternatively, one may approximate $f \in L_{\#}^{d}\left(\mathbb{T}^{d}\right)$ by trigonometric polynomials. If $f$ is a trigonometric polynomial, we may clearly obtain $Y$ as a trigonometric polynomial (after convolution). A standard limit procedure permits then to complete the argument.

\section{The equation $\operatorname{div} Y=f$ with Dirichlet condition. Proof of THEOREMS 2 AND 3}

So far we have studied problem (1.1) coupled with a periodic condition. We consider here problem (1.1) coupled with a Dirichlet condition. Usually one associates with (1.1) the "partial" Dirichlet condition

$$
Y \cdot n=0 \quad \text { on } \partial Q
$$

( $n$ is normal to $\partial Q$ ). It is quite standard that for every $f \in L_{\#}^{p}, 1<p<\infty$, there is some $Y \in W^{1, p}$ satisfying (1.1), (7.1) and

$$
\|Y\|_{W^{1, p}} \leq C\|f\|_{L^{p}}
$$

Indeed, one may look for a special $Y$ of the form $Y=\operatorname{grad} u$ and one is led to the Neumann problem

$$
\begin{cases}\Delta u=f & \text { in } Q, \\ \frac{\partial u}{\partial n}=0 & \text { on } \partial Q,\end{cases}
$$


which admits a solution $u \in W^{2, p}$ such that

$$
\|u\|_{W^{2, p}} \leq C\|f\|_{L^{p}} .
$$

It is also possible to couple problem (1.1) with the full Dirichlet condition

$$
Y=0 \quad \text { on } \partial Q
$$

For simplicity we investigate first the case where the domain is a cube and then the case of a Lipschitz bounded domain.

7.1. The case of a cube. Let $Q=(0,1)^{d}$. Here is the first result:

Theorem 2. Given $f \in L_{\#}^{p}(Q), 1<p<\infty$, there exists some $Y \in W_{0}^{1, p}(Q)$ solving (1.1) with

$$
\|Y\|_{W^{1, p}} \leq C(p, d)\|f\|_{L^{p}}
$$

where we use the standard notation

$$
W_{0}^{1, p}(Q)=\left\{Y \in W^{1, p}(Q) ; Y=0 \text { on } \partial Q\right\} .
$$

Moreover $Y$ can be chosen, depending linearly on $f$.

We will make use of the following lemma (which is a special case of Theorem 2).

Lemma 4. Given $f \in W_{0}^{1, p}(Q), 1<p<\infty$, with $\int f=0$, there exists $Y \in$ $W_{0}^{1, p}(Q)$, such that

$$
\operatorname{div} Y=f
$$

and

$$
\|Y\|_{W^{1, p}(Q)} \leq C(d)\|f\|_{W^{1, p}(Q)} .
$$

Moreover $Y$ can be chosen, depending linearly on $f$.

Proof. Following a known construction (see Adams [1, p. 58 and Nirenberg [15]), we construct $Y$ by induction on the dimension $d$. The assertion is obvious for $d=1$. Assume that it holds in dimension $(d-1)$. Let $f \in W_{0}^{1, p}\left(Q_{d}\right)$, where $Q_{d}=(0,1)^{d}$, with $\int_{Q_{d}} f=0$.

Set

$$
g\left(x^{\prime}\right)=\int_{0}^{1} f\left(x^{\prime}, t\right) d t, \text { where } x^{\prime}=\left(x_{1}, \ldots, x_{d-1}\right) \in Q_{d-1} .
$$

Clearly, $g \in W_{0}^{1, p}\left(Q_{d-1}\right)$ with

$$
\|g\|_{W^{1, p}\left(Q_{d-1}\right)} \leq C\|f\|_{W^{1, p}\left(Q_{d}\right)}
$$

and also $\int_{Q_{d-1}} g=0$. By the induction assumption there is some $Z \in W_{0}^{1, p}\left(Q_{d-1}\right)$ such that

$$
\operatorname{div}_{x^{\prime}} Z=g \quad \text { on } Q_{d-1}
$$

and

$$
\|Z\|_{W^{1, p}\left(Q_{d-1}\right)} \leq C\|g\|_{W^{1, p}\left(Q_{d-1}\right)} \leq C\|f\|_{W^{1, p}\left(Q_{d}\right)}
$$

Fix a function $\zeta \in C_{0}^{\infty}(0,1)$ such that

$$
\int_{0}^{1} \zeta(t) d t=1
$$

For $x=\left(x^{\prime}, x_{d}\right) \in Q_{d}$ set

$$
h(x)=\int_{0}^{x_{d}}\left(f\left(x^{\prime}, t\right)-\zeta(t) g\left(x^{\prime}\right)\right) d t .
$$


It is easy to see (using (7.6)) that $h \in W_{0}^{1, p}\left(Q_{d}\right)$ and

$$
\|h\|_{W^{1, p}\left(Q_{d}\right)} \leq C\|f\|_{W^{1, p}\left(Q_{d}\right)} .
$$

Moreover

$$
\frac{\partial h}{\partial x_{d}}(x)=f(x)-\zeta\left(x_{d}\right) g\left(x^{\prime}\right)
$$

Combining this with (7.5) yields

$$
f(x)=\operatorname{div}_{x^{\prime}}\left(\zeta\left(x_{d}\right) Z\left(x^{\prime}\right)\right)+\frac{\partial h}{\partial x_{d}}
$$

i.e., the conclusion holds with

$$
Y(x)=\left(\zeta\left(x_{d}\right) Z\left(x^{\prime}\right), h(x)\right) .
$$

Proof of Theorem 2. For simplicity we assume that $d=2$; the argument is similar for $d>2$.

Let

$$
Q=\left\{(x, y) \in \mathbb{R}^{2} ; \quad 0<x<1,0<y<1\right\} .
$$

Given $f \in L_{\#}^{p}(Q), 1<p<\infty$, we will construct a solution $Y \in W_{0}^{1, p}(Q)$ of (1.1); moreover

$$
\|Y\|_{W^{1, p}} \leq C_{p}\|f\|_{L^{p}}
$$

and $Y$ depends linearly on $f$. This is done in three steps.

Step 1. Construct a solution $Y \in W^{1, p}(Q)$ of (1.1) satisfying (7.7) and

$$
Y=0 \text { on the edge }\{(x, 0) ; 0<x<1\} .
$$

Proof. Set

$$
\tilde{Q}=\{(x, y) ; 0<x<1,-2<y<1\}
$$

and

$$
\tilde{f}= \begin{cases}f & \text { in } Q, \\ 0 & \text { in } \tilde{Q} \backslash Q .\end{cases}
$$

Let $Z \in W^{1, p}(\tilde{Q})$ be the solution of

$$
\operatorname{div} Z=\tilde{f} \quad \text { in } \tilde{Q}
$$

obtained via (7.2) (or via periodic conditions on $\tilde{Q}$ ).

The heart of the matter is the following construction. Write $Z=\left(Z_{1}, Z_{2}\right)$ and define $Y=\left(Y_{1}, Y_{2}\right)$ in $Q$, where

$$
\begin{aligned}
& Y_{1}(x, y)=Z_{1}(x, y)+3 Z_{1}(x,-y)-4 Z_{1}(x,-2 y), \\
& Y_{2}(x, y)=Z_{2}(x, y)-3 Z_{2}(x,-y)+2 Z_{2}(x,-2 y) .
\end{aligned}
$$

(This type of "reflection" is reminiscent of standard extension techniques in $W^{m, p}$, $m \geq 2$; see e.g. Adams [1]).

It is easy to see using $(7.9),(7,10)$ and $(7.11)$ that

$$
\operatorname{div} Y=f \quad \text { in } Q
$$

while (7.8) is clear from the definition of $Y$. 
It is important (for the next step) to observe that if we had started with the additional information

$$
Z=0 \quad \text { on the edge }\{(0, y) ;-2<y<1\} \text { of } \tilde{Q},
$$

then we could infer that $Y$ also vanishes on the edge $\{(0, y) ; 0<y<1\}$ of $Q$.

Step 2. Construct a solution $Y \in W^{1, p}(Q)$ of (1.1) satisfying (7.7) and (7.12)

$Y=0$ on the 2 adjacent edges $\{(x, 0) ; 0<x<1\}$ and $\{(0, y) ; 0<y<1\}$.

Proof. Set

$$
\hat{Q}=\{(x, y) ;-2<x<1,0<y<1\}
$$

and

$$
\hat{f}= \begin{cases}f & \text { in } Q, \\ 0 & \text { in } \hat{Q} \backslash Q .\end{cases}
$$

From Step 1 applied to $\hat{f}$ in $\hat{Q}$ we obtain a solution $\hat{Z}$ of

$$
\operatorname{div} \hat{Z}=\hat{f} \quad \text { in } \hat{Q}
$$

such that

$$
\hat{Z}=0 \quad \text { on the edge }\{(x, 0) ;-2<x<1\} \text { of } \hat{Q} .
$$

Starting with $\hat{Z}$ (instead of $Z$ ) we repeat the construction of Step 1 changing the roles of $x$ and $y$. We thus obtain a $Y \in W^{1, p}(Q)$ satisfying (1.1) in $Q,(7.7)$ and (7.12).

Step 3. Proof of Theorem 2 completed.

Consider a smooth partition of unity $\left(\theta_{i}\right), i=1,2,3,4$, subordinate to the covering of $Q$ consisting of the 4 discs of radius 1 centered at the 4 vertices. Let $Y_{i} \in W^{1, p}(Q)$ be the solution constructed in Step 2 relative to each vertex.

Set

$$
Z=\sum_{i=1}^{4} \theta_{i} Y_{i} .
$$

It is easy to see from this construction that $\theta_{i} Y_{i} \in W_{0}^{1, p}(Q), \forall i$ and thus $Z \in$ $W_{0}^{1, p}(Q)$. Moreover

$$
\operatorname{div} Z=f+\sum_{i} \nabla \theta_{i} \cdot Y_{i}
$$

and $\sum_{i} \nabla \theta_{i} \cdot Y_{i} \in W_{0}^{1, p}(Q)$. By Lemma 4 we may construct $X \in W_{0}^{1, p}(Q)$ satisfying

$$
\operatorname{div} X=\sum_{i} \nabla \theta_{i} \cdot Y_{i}
$$

and $Y=Z-X$ has all the desired properties in Theorem 2 .

Next we have a variant of Theorem $1^{\prime}$ for the full Dirichlet condition.

Theorem 3. Given $f \in L_{\#}^{d}(Q)$ there exists some $Y \in C^{0}(\bar{Q}) \cap W_{0}^{1, d}(Q)$ satisfying (1.1) with

$$
\|Y\|_{L^{\infty}}+\|Y\|_{W^{1, d}} \leq C\|f\|_{L^{d}} .
$$

Remark 10. Clearly, Theorem 3 implies Theorem $1^{\prime}$ since the function $Y$ extended by periodicity belongs to $C^{0}\left(\mathbb{T}^{d}\right) \cap W^{1, d}\left(\mathbb{T}^{d}\right)$ and satisfies (1.1) on $\mathbb{T}^{d}$. However its proof relies heavily on Theorem $1^{\prime}$. 
Proof of Theorem 3. Follow the same strategy as in the proof of Theorem 2. The only difference is that in Step 1 use Theorem $1^{\prime}$ to obtain $Z$ (instead of taking the special $Z$ in the form of a gradient). Of course the dependence of $Y$ on $f$ is not linear anymore.

In Step 3 rely on the following variant of Lemma 4 (with an identical proof).

Lemma $4^{\prime}$. Given $f \in C^{0}(\bar{Q}) \cap W_{0}^{1, p}(Q), 1<p<\infty$, with $\int f=0$, there exists $Y \in C^{0}(\bar{Q}) \cap W_{0}^{1, p}(Q)$ such that

$$
\operatorname{div} Y=f
$$

and

$$
\|Y\|_{L^{\infty}}+\|Y\|_{W^{1, p}} \leq C\left(\|f\|_{L^{\infty}}+\|f\|_{W^{1, p}}\right) .
$$

7.2. The case of Lipschitz domains. Let $\Omega$ be a Lipschitz, connected, bounded domain in $\mathbb{R}^{d}$. Recall that $\Omega$ is Lipschitz if there is a $\delta>0$ such that for every point $p \in \partial \Omega, \partial \Omega \cap B_{\delta}(p)$ is the graph of a Lipschitz function (in an appropriate coordinate system varying with $p$ ).

We have the following variants of Theorems 2 and 3.

Theorem 2'. Given any $f \in L_{\#}^{p}(\Omega), 1<p<\infty$, there exists some $Y \in W_{0}^{1, p}(\Omega)$ solving (1.1) with

$$
\|Y\|_{W^{1, p}} \leq C(p, \Omega)\|f\|_{L^{p}} .
$$

Moreover $Y$ can be chosen, depending linearly on $f$.

Theorem $3^{\prime}$. For every $f \in L_{\#}^{d}(\Omega)$ there exists some $Y \in C^{0}(\bar{\Omega}) \cap W_{0}^{1, d}(\Omega)$ solving (1.1) with

$$
\|Y\|_{L^{\infty}}+\|Y\|_{W^{1, d}} \leq C(p, \Omega)\|f\|_{L^{d}} .
$$

The heart of the argument (for both theorems) is the following.

Lemma 5. There is a bounded operator $S: L^{p}(\Omega) \rightarrow W_{0}^{1, p}(\Omega)$ such that

$$
f-\operatorname{div} S f \in W_{0}^{1, p} \quad \forall f \in L^{p}
$$

and

$$
\|f-\operatorname{div} S f\|_{W^{1, p}} \leq C\|f\|_{L^{p}} .
$$

The variant needed for the proof of Theorem $3^{\prime}$ is

Lemma $5^{\prime}$. There is a nonlinear map $S: L^{d}(\Omega) \rightarrow C^{0}(\bar{\Omega}) \cap W_{0}^{1, d}(\Omega)$ such that

$$
\|S f\|_{L^{\infty}}+\|S f\|_{W^{1, d}} \leq C\|f\|_{L^{d}}
$$

and

$$
\|f-\operatorname{div} S f\|_{W^{1, d}} \leq C\|f\|_{L^{d}} .
$$

The proof of Lemma 5 relies on the following construction. Let $Q^{\prime}$ be a cube of side $\delta$ in $\mathbb{R}^{d-1}$ and set

$$
U=\left\{\left(x^{\prime}, y\right) \in Q^{\prime} \times \mathbb{R} ; \psi\left(x^{\prime}\right)<y<\psi\left(x^{\prime}\right)+\delta\right\}
$$

where $\psi \in \operatorname{Lip}\left(Q^{\prime}\right)$. 
Lemma 6. Assume

$$
\|\nabla \psi\|_{L^{\infty}\left(Q^{\prime}\right)} \leq \varepsilon_{0}(d) \text { sufficiently small (depending only on } d \text { ). }
$$

Then, given any $g \in L^{p}(U)$ there is some $Z \in W^{1, p}(U)$ satisfying

with

$$
\begin{gathered}
\operatorname{div} Z=g \quad \text { in } U, \\
Z=0 \text { on }\left\{y=\psi\left(x^{\prime}\right) ; x^{\prime} \in Q^{\prime}\right\} \text { and on the lateral boundary of } U,
\end{gathered}
$$

$$
\|Z\|_{W^{1, p}(U)} \leq C(p, d)\|g\|_{L^{p}(U)} .
$$

Moreover $Z$ can be chosen to depend linearly on $g$.

Proof. For $x^{\prime} \in Q^{\prime}$ and $0<y<\delta$ set

$$
\tilde{g}\left(x^{\prime}, y\right)=g\left(x^{\prime}, y+\psi\left(x^{\prime}\right)\right) .
$$

Note that

where $Q=Q^{\prime} \times(0, \delta)$.

$$
\|\tilde{g}\|_{L^{p}(Q)}=\|g\|_{L^{p}(U)}
$$

By Theorem 2 there exists $\tilde{Z} \in W^{1, p}(Q)$ such that

$$
\left\{\begin{aligned}
\operatorname{div} \tilde{Z}=\tilde{g} & \text { in } Q, \\
\tilde{Z}=0 & \text { on }\left\{\left(x^{\prime}, 0\right) ; x^{\prime} \in Q^{\prime}\right\} \cup\left(\partial Q^{\prime} \times(0, \delta)\right)
\end{aligned}\right.
$$

with

$$
\|\tilde{Z}\|_{W^{1, p}(Q)} \leq C(d)\|\tilde{g}\|_{L^{p}(Q)} .
$$

Note that here $\int \tilde{g}=0$ is not required since we may consider in $\hat{Q}=Q^{\prime} \times(0,2 \delta)$ the function

$$
\hat{g}\left(x^{\prime}, y\right)= \begin{cases}\tilde{g}\left(x^{\prime}, y\right) & \text { for } x^{\prime} \in Q^{\prime} \text { and } 0<y<\delta \\ -\tilde{g}\left(x^{\prime}, y-\delta\right) & \text { for } x^{\prime} \in Q \text { and } \delta<y<2 \delta\end{cases}
$$

and then solve (using Theorem 2)

$$
\begin{aligned}
\operatorname{div} \hat{Z}=\hat{g} & \text { in } \hat{Q}, \\
\hat{Z}=Q & \text { on } \partial \hat{Q},
\end{aligned}
$$

with

$$
\|\hat{Z}\|_{W^{1, p}(\hat{Q})} \leq C(d)\|\tilde{g}\|_{L^{p}(Q)} .
$$

The restriction $\tilde{Z}$ of $\hat{Z}$ to $Q^{\prime} \times(0, \delta)$ satisfies the desired properties.

Also, it is clear by scaling that the constant in (7.21) is independent of $\delta$.

Returning to $\left(x^{\prime}, y\right) \in U$, set

$$
Z\left(x^{\prime}, y\right)=\tilde{Z}\left(x^{\prime}, y-\psi\left(x^{\prime}\right)\right)
$$

it is easy to see, using (7.18) and (7.21), that

$$
\|\operatorname{div} Z-g\|_{L^{p}(U)} \leq C(d) \varepsilon_{0}\|g\|_{L^{p}(U)}
$$

and

$$
\|Z\|_{W^{1, p}(U)} \leq C(d)\left(1+\varepsilon_{0}\right)\|g\|_{L^{p}(U)} .
$$

Choosing $\varepsilon_{0}$ such that $C(d) \varepsilon_{0}<1$ and iterating this construction yields the lemma.

The variant necessary for Theorem $3^{\prime}$ is 
Lemma 6'. Assume (7.18). Then given $g \in L^{d}(U)$ there is some $Z \in C^{0}(\bar{U}) \cap$ $W^{1, p}(U)$ satisfying (7.19), (7.20) and

$$
\|Z\|_{L^{\infty}(U)}+\|Z\|_{W^{1, d}(U)} \leq C(d)\|g\|_{L^{d}(U)}
$$

Next, we remove the smallness condition (7.18) on the Lipschitz constant of $\psi$.

Lemma 7. With the same notation as in Lemma 6, assume only that $\psi \in \operatorname{Lip}\left(Q^{\prime}\right)$.

Then, given any $g \in L^{p}(U)$, there is some $Z \in W^{1, p}(U)$ satisfying (7.19), (7.20) and

$$
\|Z\|_{W^{1, p}(U)} \leq C\left(p, d,\|\nabla \psi\|_{L^{\infty}\left(Q^{\prime}\right)}\right)\|g\|_{L^{p}(U)} .
$$

Moreover $Z$ can be chosen to depend linearly on $g$.

Proof. Consider the dilation $x^{\prime} \mapsto \tilde{x}^{\prime}=N x^{\prime}$ (only in $x^{\prime}$, not in the full $x$-variable). Set $\tilde{Q}^{\prime}=N Q^{\prime}$ and define on $\tilde{Q}^{\prime}$ the function

$$
\tilde{\psi}\left(\tilde{x}^{\prime}\right)=\psi\left(\tilde{x}^{\prime} / N\right) .
$$

Fix an integer $N$ sufficiently large so that

$$
\|\nabla \tilde{\psi}\|_{L^{\infty}\left(\tilde{Q}^{\prime}\right)}=\frac{1}{N}\|\nabla \psi\|_{L^{\infty}\left(Q^{\prime}\right)} \leq \varepsilon_{0}(d)
$$

where $\varepsilon_{0}(d)$ comes from (7.18).

Set

$$
\tilde{g}\left(\tilde{x}^{\prime}, y\right)=g\left(\frac{\tilde{x}^{\prime}}{N}, y\right)
$$

Divide the cube $\tilde{Q}^{\prime}$ (of side $N \delta$ ) into $N^{d-1}$ cubes of side $\delta$ and apply, in each of them, Lemma 6 to $\tilde{\psi}$ and $\tilde{g}$. By gluing the corresponding solutions (this is possible because all these solutions vanish on the lateral boundaries of their domains), we obtain some $\tilde{Z}\left(\tilde{x}^{\prime}, y\right) \in W^{1, p}(\tilde{U})$ satisfying

$$
\left\{\begin{aligned}
\operatorname{div} \tilde{x}^{\prime}, y=\tilde{Z}=\tilde{g} & \text { in } \tilde{U}=\left\{\left(\tilde{x}^{\prime}, y\right) \in \tilde{Q}^{\prime} \times \mathbb{R} ; \tilde{\psi}\left(\tilde{x}^{\prime}\right)<y<\tilde{\psi}\left(\tilde{x}^{\prime}\right)+\delta\right\} \\
\tilde{Z}=0 & \text { on }\left\{y=\tilde{\psi}\left(\tilde{x}^{\prime}\right) ; \tilde{x}^{\prime} \in \tilde{Q}^{\prime}\right\}
\end{aligned}\right.
$$

and the corresponding $W^{1, p}$-estimate for $\tilde{Z}$.

We now return to the variables $\left(x^{\prime}, y\right) \in U$. Write the components of $\tilde{Z}$ as

$$
\tilde{Z}=\left(\tilde{Z}^{\prime}, \tilde{Z}_{d}\right)
$$

and set

$$
Z\left(x^{\prime}, y\right)=\left(\frac{1}{N} \tilde{Z}^{\prime}\left(N x^{\prime}, y\right), \tilde{Z}_{d}\left(N x^{\prime}, y\right)\right) .
$$

It is easy to check that $Z$ satisfies all the required properties.

The variant necessary for Theorem $3^{\prime}$ is

Lemma $7^{\prime}$. With the same notation as in Lemma 6, assume only that $\psi \in \operatorname{Lip}\left(Q^{\prime}\right)$.

Then, given any $g \in L^{d}(U)$, there is some $Z \in C^{0}(\bar{U}) \cap W^{1, p}(U)$ satisfying (7.19), (7.20) and

$$
\|Z\|_{L^{\infty}(U)}+\|Z\|_{W^{1, p}(U)} \leq C\left(d,\|\nabla \psi\|_{L^{\infty}\left(Q^{\prime}\right)}\right)\|g\|_{L^{p}(U)} .
$$

We now return to the 
Proof of Lemma 5. Consider a finite covering of $\partial \Omega$ by a collection of cubes $Q_{i}, i=$ $1, \ldots, k$, of side $\delta$ such that in each $Q_{i}, \partial \Omega \cap Q_{i}$ admits a Lipschitz parametrization $\psi_{i}$. To this covering we associate functions $\theta_{0}, \theta_{1}, \ldots, \theta_{k}$ such that

$$
\begin{aligned}
& \theta_{0}+\sum_{i=1}^{k} \theta_{i}=1 \quad \text { on } \Omega, \\
& \theta_{0} \in C_{0}^{\infty}(\Omega) \text { and } \theta_{i} \in C_{0}^{\infty}\left(Q_{i}\right) \text { for } i=1, \ldots, k .
\end{aligned}
$$

Given $g \in L^{p}(\Omega)$ solve, using Lemma 7 , for $i=1,2, \ldots, k$,

$$
\left\{\begin{aligned}
\operatorname{div} Z_{i}=g & \text { in } U_{i}, \\
Z_{i}=0 & \text { on } \partial \Omega \cap Q_{i} .
\end{aligned}\right.
$$

Next solve

$$
\operatorname{div} Z_{0}=g \quad \text { in } \Omega,
$$

for example $Z_{0}=\operatorname{grad}(\Delta)^{-1}$ where $\Delta^{-1}$ is used with zero Dirichlet condition on $\partial \Omega$.

Note that

$$
Z=\sum_{i=0}^{k} \theta_{i} Z_{i} \in W_{0}^{1, p}
$$

and

$$
\operatorname{div} Z=g+\sum_{i=0}^{k} \nabla \theta_{i} \cdot Z_{i} .
$$

All the conclusions of Lemma 5 hold with

$$
S g=Z \text {. }
$$

Proof of Lemma $5^{\prime}$. We make the same construction as above, using Lemma $7^{\prime}$ in place of Lemma 7 and Theorem 2 to solve $\operatorname{div} Z_{0}=g$ in any large cube containing $\Omega$.

Theorem $2^{\prime}$ is an immediate consequence of Lemma 5 and the following general functional analysis argument applied with $E=W_{0}^{1, p}, F=L_{\#}^{p}$ and $T=$ div. (Note that $T^{*}=\operatorname{grad}$ is injective on $F^{*}=L_{\#}^{q}$, since $\Omega$ is connected.)

Lemma 8. Let $E, F$ be two Banach spaces and let $T$ be a bounded operator from E into F. Assume

$$
\begin{gathered}
N\left(T^{*}\right)=\{0\} . \\
\left\{\begin{array}{c}
\text { There is a bounded operator } S \text { from } F \text { into } E \text { and } \\
\text { a compact operator } K \text { from } F \text { into itself such that } \\
T \circ S=I+K .
\end{array}\right.
\end{gathered}
$$

Then $T$ admits a right inverse.

Proof. First we note that $T$ is onto. Indeed, in view of (7.22) it suffices to show that $T$ (or equivalently $T^{*}$ ) has closed range. This is an obvious consequence of the inequality

(which follows from (7.23)).

$$
\|f\| \leq C\left\|T^{*} f\right\|+\left\|K^{*} f\right\| \quad \forall f \in F^{*}
$$


Next, let $X$ be a complementing subspace for $N(I+K)$ in $F$ and set $Y=$ $R(I+K)$. Since $u=(I+K)_{\mid X}$ is an isomorphism onto $Y$, its inverse $u^{-1}: Y \rightarrow$ $X \subset F$ satisfies

$$
(I+K) \circ u^{-1}=I \text { on } Y .
$$

Let $Q$ be a projector from $F$ onto $Y$; since $R(I-Q)$ is finite dimensional, we may choose a base $\left(e_{\alpha}\right)$ of $R(I-Q)$ and write

$$
f=Q f+\sum_{\alpha}\left\langle e_{\alpha}^{*}, f\right\rangle e_{\alpha} \quad \forall f \in F,
$$

for some $e_{\alpha}^{*}$ 's in $F^{*}$.

Since we showed that $T$ is onto, one has, for each $\alpha$, some $\bar{e}_{\alpha} \in E$ satisfying

$$
T \bar{e}_{\alpha}=e_{\alpha} \quad \forall \alpha .
$$

Consider the operator $S_{1}: F \rightarrow E$ defined for every $f \in F$, by

$$
S_{1} f=S \circ u^{-1} \circ Q f+\sum_{\alpha}\left\langle e_{\alpha}^{*}, f\right\rangle \bar{e}_{\alpha} .
$$

Using (7.24), (7.25) and (7.26) we see that

$$
\begin{aligned}
T \circ S_{1} f & =(I+K) \circ u^{-1} \circ Q f+\sum_{\alpha}\left\langle e_{\alpha}^{*}, f\right\rangle e_{\alpha} \\
& =Q f+\sum_{\alpha}\left\langle e_{\alpha}^{*}, f\right\rangle e_{\alpha}=f
\end{aligned}
$$

for every $f \in F$. Thus $S_{1}$ is a right inverse for $T$.

Proof of Theorem $3^{\prime}$. Given $f \in L^{d}$ write, using Lemma $5^{\prime}$,

$$
f=\operatorname{div} Y_{1}+R
$$

with $Y_{1} \in C^{0}(\bar{\Omega}) \cap W_{0}^{1, d}(\Omega)$ and $R \in W_{0}^{1, d}(\Omega)$ (and the corresponding estimates).

If $\int f=0$, then $\int R=0$ and we may apply Theorem $2^{\prime}$ in any $L^{p}$ (since $\left.W^{1, d} \subset L^{p}, \forall p<\infty\right)$. In particular, if we choose $p>d$, we obtain $Y_{2} \in W_{0}^{1, p}(\Omega)$ such that

$$
R=\operatorname{div} Y_{2} .
$$

By the Sobolev imbedding, $Y_{2} \in C^{0}(\bar{\Omega})$ and $Y=Y_{1}+Y_{2}$ satisfies all the required properties.

\section{Estimation of the phase in $H^{1 / 2}+W^{1,1}$. Proof of Theorem 4}

We return in this last section to the question discussed in the Introduction concerning the control of the phase $\varphi$ in terms of $\left\|e^{i \varphi}\right\|_{H^{1 / 2}}$.

Let $\varphi$ be a smooth real-valued function on $\mathbb{T}^{d}$ and set $g=e^{i \varphi}$. The main result is the estimate

$$
\|\varphi\|_{H^{1 / 2}+W^{1,1}} \leq C(d)\left(1+\|g\|_{H^{1 / 2}}\right)\|g\|_{H^{1 / 2}} .
$$

Write $g$ as a Fourier series

$$
g=\sum_{\xi \in \mathbb{Z}^{d}} \hat{g}(\xi) e^{i x \xi}
$$


The $H^{1 / 2}$-component in the decomposition of $\varphi$ will be obtained as a paraproduct of $g$ and $\bar{g}$,

$$
P=\sum_{k}\left[\sum_{\xi_{2}} \lambda_{k}\left(\left|\xi_{2}\right|\right) \overline{\hat{g}\left(\xi_{2}\right)} e^{-i x \xi_{2}}\right]\left[\sum_{2^{k} \leq\left|\xi_{1}\right|<2^{k+1}} \hat{g}\left(\xi_{1}\right) e^{i x \xi_{1}}\right],
$$

where for each $k$ we let $0 \leq \lambda_{k} \leq 1$ be a smooth function on $\mathbb{R}_{+}$:

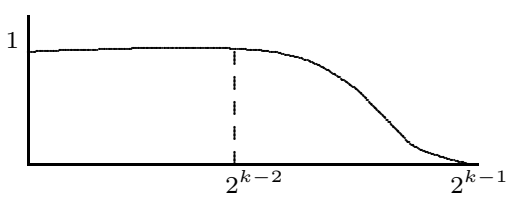

We claim that

$$
\|P\|_{H^{1 / 2}} \leq C\|g\|_{\infty}\|g\|_{H^{1 / 2}}
$$

and

$$
\left\|\varphi-\frac{1}{i} P\right\|_{W^{1,1}} \leq C\|g\|_{H^{1 / 2}}^{2} .
$$

Proof of (8.3). This is totally obvious from the construction

$$
\begin{aligned}
\|P\|_{H^{1 / 2}}^{2} & \sim \sum_{k} 2^{k}\left\|\left[\sum_{\xi_{2}} \lambda_{k}\left(\left|\xi_{2}\right|\right) \overline{\hat{g}\left(\xi_{2}\right)} e^{-i x \xi_{2}}\right]\left[\sum_{2^{k} \leq\left|\xi_{1}\right|<2^{k+1}} \hat{g}\left(\xi_{1}\right) e^{i x \xi_{1}}\right]\right\|_{2}^{2} \\
& \leq \sum_{k} 2^{k}\left\|\sum \lambda_{k}(|\xi|) \overline{\hat{g}(\xi)} e^{-i x \xi}\right\|_{\infty}^{2}\left[\sum_{|\xi| \sim 2^{k}}|\hat{g}(\xi)|^{2}\right] \\
& \leq C\|g\|_{\infty}^{2}\|g\|_{H^{1 / 2}}^{2} .
\end{aligned}
$$

Proof of (8.4). We estimate for instance

$$
\left\|\partial_{1} \varphi-\frac{1}{i} \partial_{1} P\right\|_{L^{1}}
$$

Thus, letting $\xi=\left(\xi^{1}, \ldots, \xi^{d}\right) \in \mathbb{Z}^{d}$,

$$
\partial_{1} \varphi=\frac{1}{i} \bar{g} \partial_{1} g=\sum_{\xi_{1}, \xi_{2} \in \mathbb{Z}^{d}} \xi_{1}^{1} \hat{g}\left(\xi_{1}\right) \overline{\hat{g}\left(\xi_{2}\right)} e^{i x \cdot\left(\xi_{1}-\xi_{2}\right)}
$$

and by $(8.2)$

$$
\begin{gathered}
\frac{1}{i} \partial_{1} P=\sum_{k} \sum_{2^{k} \leq\left|\xi_{1}\right|<2^{k+1}, \xi_{2}}\left(\xi_{1}^{1}-\xi_{2}^{1}\right) \lambda_{k}\left(\left|\xi_{2}\right|\right) \hat{g}\left(\xi_{1}\right) \overline{\hat{g}\left(\xi_{2}\right)} e^{i x \cdot\left(\xi_{1}-\xi_{2}\right)}, \\
\partial_{1} \varphi-\frac{1}{i} \partial_{1} P=\sum_{k} \sum_{2^{k} \leq\left|\xi_{1}\right|<2^{k+1}, \xi_{2}} m_{k}\left(\xi_{1}, \xi_{2}\right) \hat{g}\left(\xi_{1}\right) \overline{\hat{g}\left(\xi_{2}\right)} e^{i x \cdot\left(\xi_{1}-\xi_{2}\right)},
\end{gathered}
$$

where by definition of $\lambda_{k}$

$$
m_{k}\left(\xi_{1}, \xi_{2}\right)=\xi_{1}^{1}-\lambda_{k}\left(\left|\xi_{2}\right|\right)\left(\xi_{1}^{1}-\xi_{2}^{1}\right)= \begin{cases}\xi_{2}^{1} & \text { if }\left|\xi_{2}\right| \leq 2^{k-2} \\ \xi_{1}^{1} & \text { if }\left|\xi_{2}\right| \geq 2^{k-1}\end{cases}
$$


Estimate

$$
\left\|\partial_{1} \varphi-\frac{1}{i} \partial_{1} P\right\|_{1} \leq \sum_{k_{1}, k_{2}}\left\|\sum_{\left|\xi_{1}\right| \sim 2^{k_{1}},\left|\xi_{2}\right| \sim 2^{k_{2}}} m_{k_{1}}\left(\xi_{1}, \xi_{2}\right) \hat{g}\left(\xi_{1}\right) \overline{\hat{g}\left(\xi_{2}\right)} e^{i x \cdot\left(\xi_{1}-\xi_{2}\right)}\right\|_{1}
$$

Distinguish the contributions of

$$
\sum_{k_{1} \sim k_{2}}+\sum_{k_{1}<k_{2}-4}+\sum_{k_{1}>k_{2}+4}=(8.12)+(8.13)+(8.14) .
$$

Clearly $2^{-k} m_{k}\left(\xi_{1}, \xi_{2}\right)$ restricted to $\left[\left|\xi_{1}\right| \sim 2^{k}\right] \times\left[\left|\xi_{2}\right| \sim 2^{k}\right]$ is a smooth multiplier satisfying the usual derivative bounds. Therefore

$$
(8.12) \leq C \sum_{k} 2^{k}\left\|\sum_{\left|\xi_{1}\right| \sim 2^{k}} \hat{g}\left(\xi_{1}\right) e^{i x \xi_{1}}\right\|\left\|_{2}\right\|_{\left|\xi_{2}\right| \sim 2^{k}} \hat{g}\left(\xi_{2}\right) e^{i x \xi_{2}}\left\|_{2} \sim\right\| g \|_{H^{1 / 2}}^{2} .
$$

If $k_{1}<k_{2}-4$, then $\left|\xi_{2}\right|>2^{k_{1}}$ and $m_{k_{1}}\left(\xi_{1}, \xi_{2}\right)=\xi_{1}^{1}$ by (8.10). Therefore

$$
\begin{aligned}
& (8.13)=\sum_{k_{1}<k_{2}-4}\left\|\sum_{\left|\xi_{1}\right| \sim 2^{k_{1}},\left|\xi_{2}\right| \sim 2^{k_{2}}} \xi_{1}^{1} \hat{g}\left(\xi_{1}\right) \overline{\hat{g}\left(\xi_{2}\right)} e^{i x \cdot\left(\xi_{1}-\xi_{2}\right)}\right\|_{1} \\
& \leq \sum_{k_{1}<k_{2}-4} 2^{k_{1}}\left\|\sum_{\left|\xi_{1}\right| \sim 2^{k_{1}}} \hat{g}\left(\xi_{1}\right) e^{i x \xi_{1}}\right\|_{2} \cdot\left\|\sum_{\left|\xi_{2}\right| \sim 2^{k_{2}}} \hat{g}\left(\xi_{2}\right) e^{i x \xi_{2}}\right\|_{2} \\
& \leq \sum_{k_{1}<k_{2}} 2^{k_{1}}\left(\sum_{\left|\xi_{1}\right|<2^{k_{1}}}\left|\hat{g}\left(\xi_{1}\right)\right|^{2}\right)^{1 / 2}\left(\sum_{\left|\xi_{2}\right| \sim 2^{k_{2}}}\left|\hat{g}\left(\xi_{2}\right)\right|^{2}\right)^{1 / 2} \leq C\|g\|_{H^{1 / 2}}^{2} \text {. }
\end{aligned}
$$

If $k_{1}>k_{2}+4$, then $\left|\xi_{2}\right|<2^{k_{1}-2}$ and $m_{k_{1}}\left(\xi_{1}, \xi_{2}\right)=\xi_{2}^{1}$ and the bound on (8.14) is similar.

\section{REFERENCES}

[1] R.A. Adams, "Sobolev spaces", Acad. Press, 1975. MR 56:9247

[2] D.N. Arnold, L.R. Scott and M. Vogelius, Regular inversion of the divergence operator with Dirichlet boundary condition on a polygon, Ann. Sc. Norm. Pisa, Serie IV, 15 (1988), 169192. MR 91i:35043

[3] J. Bourgain, H. Brezis and P. Mironescu, Lifting in Sobolev spaces, J. d'Analyse 80 (2000), 37-86. MR 2001h:46044

[4] On the structure of the Sobolev space $H^{1 / 2}$ with values into the circle, C. R. Acad. Sc. Paris 331 (2000), 119-124. MR 2001m:46068

[5] $\longrightarrow$, in preparation.

[6] H. Brezis, "Analyse fonctionnelle, théorie et applications", Masson, 1983. MR 2001m:46068

[7] D. Burago and B. Kleiner, Separated nets in Euclidean space and Jacobians of bi-Lipschitz maps, Geom. Funct. Anal. 8 (1998), 273-282. MR 99d:26018

[8] B. Dacorogna and J. Moser, On a partial differential equation involving the Jacobian determinant, Ann. Inst. H. Poincaré, Anal. Nonlinéaire, 7 (1990), 1-26. MR 91i:58148

[9] G. Duvaut and J.L. Lions, "Les inéquations en mécanique et en physique", Dunod, 1972; English translation, Springer, 1976. MR 57:4778

[10] C. Fefferman and E. Stein, $H^{p}$ spaces of several variables, Acta Math. 129 (1972), 137-193. MR 56:6263

[11] M. Gromov, Asymptotic invariants of infinite groups, in "Geometric Group Theory", Vol.2 (G. A. Niblo, M. A. Roller, eds.), Cambridge Univ. Press, 1993. MR 95m:20041

[12] E. Magenes and G. Stampacchia, I problemi al contorno per le equazioni differenziali di tipo ellitico, Ann. Sc. Norm. Pisa 12 (1958), 247-357. MR 23:A1140

[13] C. T. McMullen, Lipschitz maps and nets in Euclidean space, Geom. Funct. Anal. 8 (1998), 304-314. MR 99e:58017 
[14] J. Nečas, Sur les normes équivalentes dans $W_{p}^{(k)}(\Omega)$ et sur la coercitivité des formes formellement positives, in "Equations aux dérivées partielles", Presses de l'Université de Montreal, 1966.

[15] L. Nirenberg, "Topics in nonlinear functional analysis", New York Univ. Lecture Notes, 197374. MR 58:7672

[16] D. Ornstein, A non-inequality for differential operators in the $L_{1}$ norm, Arch. Rat. Mech. Anal. 11 (1962), 40-49. MR 26:6821

[17] $\mathrm{T}$. Rivière and $\mathrm{D}$. Ye, Une résolution de l'équation à forme volume prescrite, $\mathrm{C}$. $\mathrm{R}$. Acad. Sc. Paris 319 (1994), 25-28. MR 95f:35055

[18] _ Resolutions of the prescribed volume form equations, Nonlinear Differential Equations Appl. 3 (1996), 323-369. MR 97g:35045

[19] J.L. Rubio de Francia, A Littlewood-Paley theorem for arbitrary intervals, Rev. Mat. Iberoamericana 1 (1985), 1-14. MR 87j:42057

[20] R. Temam, "Navier-Stokes equations", North-Holland, revised edition, 1979. MR 82b:35133

[21] A. Uchiyama, A constructive proof of the Fefferman-Stein decomposition of BMO $\left(\mathbb{R}^{n}\right)$, Acta Math. 148 (1982), 215-241. MR 84h:42037

[22] X. Wang, A remark on the characterization of the gradient of a distribution, Applic. Anal. 51 (1993), 35-40. MR 95k:46064

[23] R. Wojtaszczyk, "Banach spaces for analysts", Cambridge Univ. Press, 1991. MR 93d:46001

[24] D. Ye, Prescribing the Jacobian determinant in Sobolev spaces, Ann. Inst. H. Poincaré, Anal. Nonlinéaire 11 (1994), 275-296. MR 95g:35058

Institute for Advanced Study, Princeton, New Jersey 08540

E-mail address: bourgain@math.ias.edu

Analyse Numérique, Université P. et M. Curie, B.C. 187, 4 Pl. Jussieu, 75252 Paris Cedex 05, France

E-mail address: brezis@ccr.jussieu.fr

Current address: Department of Mathematics, Rutgers University, Hill Center, Busch Campus, 110 Frelinghuysen Rd., Piscataway, New Jersey 08854

E-mail address: brezis@math.rutgers.edu 